University of Warwick institutional repository: http://go.warwick.ac.uk/wrap This paper is made available online in accordance with publisher policies. Please scroll down to view the document itself. Please refer to the repository record for this item and our policy information available from the repository home page for further information.

To see the final version of this paper please visit the publisher's website. Access to the published version may require a subscription.

Author(s): JAMES S. ADELMAN and GORDON D. A. BROWN

Article Title: Methods of testing and diagnosing model error: Dual and single route cascaded models of reading aloud

Year of publication: 2008

Link to published version: http://dx.doi.org/10.1016/j.jml.2007.11.008

Publisher statement: None 
Adelman, J. S., \& Brown, G. D. A. (2008). Methods of testing and diagnosing model error: Dual and single route cascaded models of reading aloud. Journal of Memory and Language, 59, 524-544.

www.elsevier.com/locate/jml

DOI: $10.1016 / j \cdot j m 1.2007 .11 .008$ 
Running head: ANALYZING MODEL ERROR IN THE DRC

Methods of Testing and Diagnosing Model Error:

Dual and Single Route Cascaded Models of Reading Aloud

James S. Adelman and Gordon D. A. Brown

University of Warwick

12,292 words.

Corresponding Author:

James S. Adelman

Department of Psychology,

University of Warwick,

Gibbet Hill Road,

COVENTRY,

CV4 7AL,

UK.

Telephone: +44 (0) 2476150233

Electronic mail: J.S.Adelman@warwick.ac.uk 


\begin{abstract}
Models of visual word recognition have been assessed by both factorial and regression approaches. Factorial approaches tend to provide a relatively weak test of models, and regression approaches give little indication of the sources of models' mispredictions, especially when parameters are not optimal. A new alternative method, involving regression on model error, combines these two approaches with parameter optimization. The method is illustrated with respect to the dual route cascaded model of reading aloud. In contrast to previous investigations, this method provides clear evidence that there are parameter-independent problems with the model, and identifies two specific sources of misprediction made by model.
\end{abstract}




\section{Methods of Testing and Diagnosing Model Error: Dual and Single Route Cascaded Models of Reading Aloud}

The ideal of modeling cognitive processes involves testing the consequences of the processes conjectured in models against behavioral data. In the context of visual word recognition, where many of the relevant predictors are properties of words, two approaches have emerged to testing such models. The first is a factorial approach in which the question is whether a model can simulate some set of empirically obseved significant effects. The second is a regression approach in which the regression performance (correlation with data) of the model's behavior is compared to a regression equation with the relevant predictors.

Coltheart, Rastle, Perry, Langdon, and Ziegler (2001) applied both approaches to the assessment of their dual route cascaded (DRC) model of reading aloud. This model incorporates a lexical (vocabulary-based) interactive-activation (IA) route and a nonlexical (spelling-sound rule-based) route that interact (collaborate or compete) to generate pronunciations. They argued, primarily on the basis of the results of the factorial approach, that "the DRC model is the most successful of current computational models of reading" (p. 251). They also argued that "if there is no other theory that ... [is] both complete and sufficient, resting on laurels is a reasonable thing to do until the emergence of a competitor." (p. 204). It is our contention that neither the factorial approach nor the regression approach nor their combination, as currently applied, is adequate to demonstrate that a model is sufficient to explain effects or data. One reason is that the parameters - numerical values defined to control the operation of a model without a fully specified relationship with independent variables — used in such analyses are not chosen to be optimal for the data set in question; in consequence, the criterion for sufficiency is artificially low to compensate. 
In this paper, we (i) set out the problems with the existing approaches; (ii) introduce a method of combining the two approaches with optimal parameter selection that resolves several of these problems; (iii) describe the DRC model and our optimization of its parameters for the Spieler and Balota (1997) data set, which was among those examined by Coltheart et al. (2001); (iv) demonstrate the theoretical leverage the approach can obtain with the application of the technique to the DRC; and (v) discuss issues surrounding the analyses, including the data collection strategies that support the method. We choose the DRC model for this exposition for three reasons. First, this model is a complete model of reading aloud that appears to be able to account for a relatively wide range of effects, indeed one that is wider than other models, and thus is in need of strict testing. Second, the model is relatively well-suited to the approach described here. Finally, Coltheart et al. have argued against using the optimization of parameters and in favor of a pure factorial approach, despite some examination of regression approach results.

\section{The Factorial Approach}

The factorial approach to deciding whether a model is adequate to explain a particular cognitive process is to generate a list of effects (including interactions) that one has reason to believe occur in the task of interest, and then determine whether the model can predict the direction of these effects correctly.

Coltheart et al. (2001), for instance, list 18 effects in word naming for which their model can account, and of these, six relate to a standard word naming task (i.e., involve neither nonwords nor priming): (i) regularity, (ii) frequency, (iii) their interaction, (iv) position of (first) irregularity, (v) rime consistency, and (vi) length. One further effect in standard word naming that Coltheart et al. attempt to account for with their model is the orthographic neighborhood size ( $N$; Coltheart, Davelaar, Jonasson, \& Besner, 1977; Andrews, 1989, 1992) effect. They find that the model with their standard parameters 
does not predict an effect of orthographic $N$, but that an alteration to one of the parameters causes the model to predict such an effect. Thus it was demonstrated that the DRC can predict an orthographic $N$ effect.

\section{Suboptimal Parameter Sets.}

This change in parameters to account for orthographic $N$ effects raises the first problematic issue for the factorial approach: Whether and when it is appropriate to simulate each data set with different parameters. Coltheart et al.'s (2001) assert that they "would in any case not be interested in an approach in which each set of human data is simulated with a different set of DRC parameters" (p. 218). However, the DRC model is supposed (through parameter changes) to account for (i) strategic effects that are caused by changes in the stimulus list (see also Rastle \& Coltheart, 1999) and (ii) individual differences (arising from the strengths of the different routes). Since each set of human data presumably comes from a different experiment with different items and participants, it is hard to see why the parameters should not be different. Indeed, we would argue that if one is following the good practice of seeking to disconfirm proposed theories, it is necessary to optimize parameters in order to avoid the contention that the chosen parameters are at fault, rather than the mechanisms of the model under test.

\section{Incompleteness of Data.}

A corollary of the specificity of parameters to experiments is that the results of any given experiment speak only to the existence of some pattern under the specific conditions (and hence parameters) of that experiment, and not to those of another experiment. For instance, Coltheart et al. (2001) suggest that "it will be necessary to study whether the DRC model can yield a beneficial effect of $N$ on word naming through a modification of the parameter set that does not compromise its successful simulation of the other effects" (pp. 224-225). This suggestion requires the implicit assumption that $N$ and position of 
irregularity effects will occur together in one experiment, and their model should account for such a pattern. However, whether such an account is in fact desirable depends on a number of empirical questions because these effects have previously been demonstrated in different experiments, with different items and different participants. This means the effects could be compatible with two different parameterizations of the correct model. That is, in the absence of a data set demonstrating the combined pattern, it might yet be the case that these effects are mutually exclusive, and a model could be correct to predict them only with different parameter sets. Whilst this particular example could be resolved with an experiment with both position of irregularity and $N$ as factors, any single such experiment would not solve the problem for other pairs of predictors, nor triples, quadruples and so on. Factorial designs with few factors will thus lead to ambiguities as to whether sets of effects should co-occur with the same parameters. Attempts to include more factors will include increasingly many items if the experiments are not to lose all their power. These items will necessarily be less well controlled (see Item Selection below), the analysis will tend therefore to rely on statistical partialing (i.e., regression) — and note that has already been the case for at least one study of the main effect of position of irregularity (Rastle \& Coltheart, 1999) — and generally increasingly resemble the regression and mega-study approach.

\section{Ignorance of Effect Magnitude.}

The intercept and slope of the linear equation linking model cycles (response latency in the model) to observed experimental response latencies are parameters that often change in a hidden fashion when the factorial approach is used. Although such changes will not change the significance of any effect in the model, the fit of the model should be considered somewhat suspect if, say, (in a single experiment) a significant 10ms effect of one variable corresponds to significant two cycle difference in the model, and a significant $10 \mathrm{~ms}$ effect of another variable corresponds to a significant 20 cycle difference in the 
model (cf. Norris, 1994; Reynolds \& Besner, 2004; Besner \& Borowsky, 2006).

\section{Difficulty of Detecting Effects.}

It is anyway the case that the set of significant effects for a correct model is not necessarily the same as that for the data, as model predictions are often relatively stable measures of central tendency. This occurs when the argument is made that the model predicts an effect that appears absent in data. For example, Siakaluk, Sears, and Lupker (2002) and Sears, Campbell, and Lupker (2006) have criticized the multiple read-out model (Grainger \& Jacobs, 1996) for predicting an inhibitory effect of higher-frequency neighbors that is not observable with stimuli in English. The problem arises because (as will be shown below in the case of the DRC) model predictions have smaller standard errors than data, and so conventional tests have more power to detect effects in models. Using the combination of a significance test on the model and another on the data may therefore lead to a liberal criterion for rejecting models because a difference in significance need not correspond to a difference in magnitude of effect. Frost, Kugler, Deutsch, and Forster (2006) have, for instance, criticized models with alphabetic lexical organization for predicting facilitatory priming from orthographic neighbours when 12 experimental conditions (across seven experiments) yielded no significant facilitation (although the mean effect across these 12 conditions was $6.5 \mathrm{~ms}$ with $S E 0.66$ ).

One can only be confident that inferenece on the basis of the results of such a pair of significance tests will not lead to error is where both tests are significant with different sign of effect. Rastle and Coltheart (1998), for instance, rejected the DRC-G (a variation of the DRC with the nonlexical route timed by whole graphemes rather than letters) because it predicted faster responses for stimuli containing multi-letter graphemes, and slower responses were empirically observed with such a manipulation (on nonwords). Relying solely on such cases would be hyper-conservative on three counts: (i) only mispredictions of sign can be detected; (ii) the detection does not make use of the 
(item-by-item) paired nature of prediction and data; and (iii) the implied solution to the Behrens-Fisher (heterogeneity of variance) problem has size (Type I error rate) distortion due to overestimation of the standard error.

\section{Item Selection.}

A related issue in applying the factorial approach to psycholinguistic tasks is in selecting stimuli to realize contrasts of theoretical interest. It is at best difficult to select words that differ only on the lexical variable(s) of interest, and not on some other variable, especially for variables that are highly correlated (Cutler, 1981). This exacerbates the difficulty that one cannot know whether one's list of effects is complete, as comparisons based on few items lack power in by-items analyses. Moreover, one can only know about a failure to fit the data that is related to absence or presence of effects that have been designed into the experiment. Poor predictions for particular words will not be distinguishable from chance variation. It is not possible to identify a set of words included in the experiment for which the model does particularly badly, unless it is due to a contrast of interest designed into the experiment. Despite the modeler specifying quantitatively all the predictions of the model, it is still necessary for an experimenter testing the model to have some degree of analytic knowledge regarding the qualitative predictions of the model in order to generate a useful design. Therefore, existing data from factorial experiments have limited capability to guide new hypothesis-generation.

\section{Summary of Problems with the Factorial Approach.}

In summary, the factorial approach offers the promise of rejecting models when they do not accommodate observable effects and allows the direct evaluation of whether a model proper can explain effects via confound, but in its application to models proper, (i) the argument is often available that parameter changes might correct the apparent misfit; (ii) it is often desirable to make assumptions about the results of predictor combinations 
that have not occurred in factorial designs; (iii) relative magnitude or importance of effects is not usually tested or testable; (iv) the method is hypersensitive in that it may reject models that predict effects that are present but difficult to detect in data (because of low power); (v) confounds are hard to avoid (although this is mitigated by the fact that a correct model will correctly predict the results of a confounded experiment anyway); and (vi) the list of effects may be incomplete, even in relation to collected data, meaning that success for all the known effects does not necessarily correspond to accounting for the data.

\section{The Regression Approach}

The regression approach, by contrast, evaluates correlations between model predictions and data on an item-by-item (word-by-word) basis. The worst case for a model is when its predictions fail to significantly correlate with the data. Besner, Twilley, McCann, and Seergobin (1990) described cases where this is true of the Seidenberg and McClelland (1989) model, for instance. A model's correlation can also be compared to the multiple correlation of a number of variables of interest: If the model's correlation is smaller, then it cannot be explaining the effect of all the variables in the regression model. Spieler and Balota (1997) examined the models of Seidenberg and McClelland and Plaut, McClelland, Seidenberg, and Patterson (1996) on such a basis, and found that they do not account for all the effects of log frequency, length and $N$. Besner (1999) also examined the performance of these two models as well as the lexical route of the DRC on the basis of naming latencies for 300 words; he drew similar conclusions for the Seidenberg and McClelland and Plaut et al. models, and found that the DRC's lexical route came close to accounting for the same amount of variance as these three effects, accounting for approximately $13 \%$ of the variance, where these effects accounted for $15 \%$. 
Coltheart et al. (2001) assessed their DRC model in the same fashion against the Spieler and Balota (1997) data set, among others. They found that the predictions of the model could account for only $3.49 \%$ of the variance in this human naming latency data set. It therefore appeared that the DRC model also does not quantitatively account for all the effects of log frequency, length and $N$, as these three variables together can account for in excess of $20 \%$ of the variance in these data. Again, it is of course perfectly reasonable to assume that another set of parameters would yield improved prediction, as the parameters used were chosen by hand. Indeed, we will show below that this is the case. It is plausible that changing the parameter set might recalibrate the strengths of these effects, which appear to be present according to the factorial approach, so that the model can simultaneously account for their effects in the correct proportions.

\section{Absent Effects.}

Nevertheless, even were there continued subcriterial fit after parameter optimization, it would be essentially impossible to determine from this type of regression (alone) where precisely the model is at fault. One reason is that the locus of fault need not be among the predictors in the regression. If the model predicts an effect that is absent in the data this will cause shrinkage of the observed proportion of variance for which the model accounts, since a greater proportion of the model's variance is due to this apparent noise (apparent, because it is in fact bias). For instance, suppose that the only factor affecting some lexical task were word frequency, but we had a model that (by some non-transparent mechanism) predicted not only an effect of word frequency but also a strong effect of length. Such a model could readily be shown to correlate poorly with response times, and indeed more poorly than word frequency alone. The inclusion or not of length as a predictor in the regression on the data would not affect these results, and its inclusion would be contraindicated by a non-significant effect. Nevertheless, it would be the length effect that is the critical aspect of the model behavior that should be 
recognized as faulty. As with the factorial approach, analytic model knowledge is useful. Moreover, if one looks at the error in prediction of individual words, it is unclear which of the large errors are due to noise and which are consistently misestimated, so the data do not give direct guidance in finding these absent effects.

\section{Absent Variables.}

Moreover, many more factors can be added to regressions on Spieler and Balota's (1997) data, as Balota, Cortese, Sergent-Marshall, Spieler, and Yap (2004) have done to assess several effects, but comparing total variance accounted for by the regression to that by the psychological model would only complicate the problem of finding where a model does and does not succeed. Further, even were some model to exceed the criterial proportion of variance for which it is known to be necessary to account, this does not imply that there is not serious misfit for any of the items in the data, only that the misfit is less than that for the known predictors. The criterion for misfit would nevertheless be stricter than with the factorial approach.

\section{Model-Specific Analyses.}

One way that Coltheart et al. (2001) attempted to use the regression approach to identify possibly useful modifications to the DRC model is a comparison of the quality of its predictions for words and nonwords for the data of Weekes (1997). The model makes considerably better predictions for the nonwords than the words of Weekes' experiment. Coltheart et al. reasoned that this is indicative of problems in the lexical route, which uses word-specific information, rather than the nonlexical route, which processes stimuli using spelling-sound correspondences, since the lexical route contributes to word naming, but less to nonword naming. This pattern does not necessarily indicate that the nonlexical route operates entirely correctly for nonwords, let alone for words, and indeed for words, it will be seen that it probably does not. Moreover, such comparisons can only be valid in 
the presence of well-matched stimuli, incurring some of the problems of the factorial approach. Even were this the case here, as a methodology for theory testing, this type of analysis cannot be general, since few models truly operate in the necessary gated fashion.

Summary of Problems with the Regression Approach.

In summary, the regression approach offers the promise of rejecting psychological models when they perform less well than simple regression models based on observable effects. However, (i) any failure may be attributed to suboptimal parameters; (ii) the version of this approach discussed so far (as used by Besner et al., 1990; Spieler \& Balota, 1997; Balota \& Spieler, 1998; Coltheart et al., 2001) does not permit localization of problematic predictions; (iii) the criterion derived from any list of effects is too low if the list of effects is incomplete; and (iv) strategies such as comparing regressions for different item groups are applicable in model-specific cases that occur rarely if at all.

An Approach to Diagnosing Models

\section{Optimized Parameters.}

Can the problems of the factorial and regression approaches, as they are currently applied, be rectified? The changes that are needed are not revolutionary. The "optimal parameters" problem — that better parameters might rectify any apparent misfit — is common to both approaches and is a severe obstacle to progress via theory refutation or refinement, but has an obvious solution. Although optimizing parameters for complex models, such as the DRC, is computationally expensive, doing so will necessarily overcome this artificial barrier to falsification.

A theoretical objection to optimizing parameters made by Coltheart et al. (2001) is that if performed on a case-by-case (experiment-by-experiment) basis, then one may obtain different parameters to explain different effects, even if these effects co-occur. As we have pointed out, whether or not the effects co-occur and with what relative 
magnitudes are empirical issues. They can be resolved by considering a larger dataset that can be used to evaluate both, or preferably, all and any effects of interest. Then analyses can be performed on both model and data to identify predicted and actual effects. Where a failure for the model to simulate the data occurs, it cannot be due to incorrect parameters, as any change in the parameters necessarily will be at the detriment of some other aspect of the fit, to the extent that the overall fit to the data is relatively compromised. Moreover, availability of higher powered technology can overcome technical difficulties that might prevent such optimization.

\section{Regressing Model Predictions.}

So, if optimal parameters are chosen, it becomes possible with relative certainty to detect some levels of failure using the regression approach, when the fit (e.g., $R^{2}$ ) of the model does not reach the levels of a regression with known factors. Loci of failure can then be identified using a combination of the factorial and regression approaches: From the factorial approach, we take the idea of treating the model predictions in a manner comparable to that of the data, but in the manner of the regression approach, the entire set of data is analysed, with the variables that would otherwise have been experimentally controlled entered as covariates. Such analyses are much more powerful than those created by selection of items, as they use more data points, and discard fewer data (because they avoid the imprecision of creating discrete categories). This is especially important to counteract the reduction in power due to variance inflation in cases where highly correlated predictors are considered, and such cases can be theoretically important. A further important advantage is that unusual or unexpected contrasts may also be tested without the difficulties of selecting novel controlled stimuli.

The use of a regression model to examine the fit of the model to the data also helps resolve the issue of the relative magnitudes of the effects. Not only will an inability of the model to reproduce the correct relative magnitudes of the effect cause the overall fit to be 
inferior, but examination of the regression coefficients could reveal which effects are relatively too strong or too weak in the predictions.

\section{Regressing Model Error.}

In addition to the point that effect magnitude is important in identifying problematic predictions, a key issue in comparing a psychological model to data is that the model may predict a significant effect that is not significant in the data, but only due to differences in power. The proposed approach addresses both of these problems directly by examining the regression equations for the difference between the model predictions and the data points, that is, the model's error. If the model and data regression coefficients differ, and a factor is a significant predictor of model error, then we can infer that the model is wrong in some aspect associated with this factor (although not necessarily directly). Moreover, localization of problems to a specific aspect of the data may not localize them to a specific, readily modified, aspect of the model, as changes to the model could greatly affect the optimal parameters in the unchanged components of a model, due to an alteration in the optimal division of labor. If several problems are indicated, they may all share a single root cause in the structure of the model. A direct modification to resolve one such problem might then simultaneously solve the other problems. However, a targeted modification to another part of the model to solve a different problem might only introduce more problems. Nevertheless, some indication of how the model fails to capture the data will be of use in determining future theoretical steps.

\section{The Illustration of the Technique}

We will now proceed to demonstrate the proposed new approach with reference to the Coltheart et al. (2001) DRC model. An alternative single-route (SRC) model that consists only of the lexical route (unchanged from the DRC) is also used; this allows us to form a model-specific test of the necessity of grapheme-phoneme conversion rules to 
augment interactive-activation (IA) models of this kind.

\section{The Dual Route Cascaded Model}

The DRC model is a computational instantiation of dual route theory by Coltheart et al. (2001) involving some assumptions that are unique to versions of this theory associated with Coltheart and colleagues. Its lexical route is an extended (horizontally generalized) and modified version of the IA model of McClelland and Rumelhart (1981). This is supplemented by a non-lexical all-or-nothing grapheme-phoneme conversion (GPC) rule route. We will briefly describe the operation of the DRC model; readers are referred to the original paper for a more complete explanation. Where some aspects of its operation are not described in the original paper, these have been reverse-engineered from the operation of the implementation of the model that Coltheart et al. have made available.

\section{Feature Level}

The feature level consists of 8 sets of units of the type described by Rumelhart and Siple (1974); each set consists of 14 pairs of feature-present and feature-absent units. In each pair, if the feature is present, the feature-present unit takes the value one, and the feature-absent unit the value zero, with the pattern reversed if the feature is absent. They are not influenced by the activations of any other units, only by the stimulus presented to the model, and they are the only component of the model directly influenced by the stimulus. Words are left-justified when presented to the model.

\section{Letter Level}

The letter level consists of 8 sets of 27 units representing the 26 letters of the English alphabet and one extra for "absent". The activation of units in this level, $a_{i}(t)$ for unit $i$ on time tick $t$ is governed by Equation 1:

$$
a_{i}(t+1)=\left(1-\theta_{i}\right) a_{i}(t)+\rho \epsilon_{i}(t)
$$


subject to the constraint that $a_{i}$ must be in $[0,1]$ (and takes the nearer value when it falls outside this range), where $\theta_{i}$ is the (positive) letter decay parameter (a decay parameter exists at each level), $\rho$ is a global activation parameter, and $\epsilon_{i}(t)$ is the squashed net input to the unit $i$. The squashed input $\epsilon_{i}(t)$ is calculated from the net input $n_{i}(t)$ thus:

$$
\epsilon_{i}(t)= \begin{cases}n_{i}(t)\left(1-a_{i}(t)\right), & n_{i}(t) \geq 0 \\ n_{i}(t) a_{i}(t), & n_{i}(t)<0\end{cases}
$$

where $n_{i}(t)$ is calculated as the sum of the products of the activations of connected units at $t-1$ with the relevant connection weight parameters. Net inputs to the letter level units are influenced by feature level units that are consistent with the letter units per the feature-letter excitation parameter, and those that are inconsistent per the feature-letter inhibition parameter. Net input is also influenced by the orthographically consistent and inconsistent words with the orthography-letter excitation and orthography-letter inhibition parameters. Moreover, every letter within each set gives inhibition to every other letter within the same set according to the letter-letter inhibition parameter.

\section{Orthographic Lexicon}

The orthographic lexicon consists of a unit for every orthographic word form in the model's vocabulary. Their activations and net inputs are governed by Equations 1 and 2 . Each unit receives activation from consistent letter units and is inhibited by inconsistent ones, according to the letter-orthography excitation and letter-orthography inhibition parameters. Consistent phonology units also give excitation in proportion to the phonology-orthography excitation parameter. Further, orthographic units are mutually inhibitory, per the orthographic-orthographic inhibition parameter. Lower-frequency words are additionally inhibited by an additional bias to the net input of value $\left(\frac{\lg f_{i}}{6.068}-1\right) \phi$ where $f_{i}$ is the frequency of the word $i$, and $\phi$ is the frequency scaling parameter. 6.068 is slightly more than the Napierian logarithm of the highest frequency in the lexicon ${ }^{1}$. 


\section{Grapheme-Phoneme Conversion Rule System}

The grapheme-phoneme rule system consists of 8 sets of 44 units, each corresponding to a phoneme, or "absent", in a left-justified phonological representation. The activation of these units is governed by the activation of the most activated units within each of the sets of letter units on the $s a m e^{2}$ time tick. On any given time tick, only

a subset of the letters are considered: The first (left-most) $\left\lfloor 1+\frac{t-\alpha}{\beta}\right\rfloor$ letters are considered (if positive), where $\alpha$ is the GPC delay parameter and $\beta$ is the GPC rate parameter.

Phoneme units are activated from the letter units according to a set of GPC rules derived from analyses of the vocabulary of the model. These operate from left-to-right on the input to produce left-to-right output. Rules may be selectively operational at the beginning of the input, at the end of the input, which is detected when the to-be-processed letters are followed by the absent letter, or in the middle of the input (i.e., anywhere else in the input), or a combination of two, or operational in every position.

Once a set of letters has contributed a phoneme or phonemes to the output, consideration proceeds to the following letter(s). Some contextual rules operate only in certain (preceding or following) contexts, but the contextual letter(s) are not part of the rule, and are not passed over for consideration if they are following context. Other distant rules (all of the "silent $e$ " type) are applied in response to letter patterns that contain a central portion that can be matched by any letter; this wildcard letter is not considered part of the rule, but those letters after this are part of the rule (and therefore affect the weighting discussed below). Consideration passes to the wildcard letter, and those letters thereafter are not passed over for consideration. Any remaining "absent" letters that are available for consideration at this stage are converted to final "absent" phonemes.

The strength of the activation of the output in any position in the rule system is the arithmetic mean activation of the letters that form part of the rule generating the phoneme there at this stage. Finally, a set of search-and-replace phonotactic rules are 
applied to the output; most of these apply to the phoneme $/ s /$ in post-vocalic contexts, but only when produced by the rule $\mathrm{S} \rightarrow / s /$, and in particular not when produced by the rule $\mathrm{SE}_{\text {final }} \rightarrow / s /$ (e.g., in HOSE).

\section{Phonological Lexicon}

The phonological lexicon consists of a unit for every phonological word form in the model's vocabulary. The activation and net input are again governed by Equations 1 and 2. Excitatory input comes from consistent orthographic word units and consistent phoneme units according to orthography-phonology excitation and phoneme-phonology excitation parameters. Inhibitory input comes from inconsistent phoneme units per the phoneme-phonology inhibition parameter, and from other phonology units with the phonology-phonology inhibition parameter. The phonological lexicon is also subject to the same frequency-based bias to input as the orthographic lexicon; in Coltheart's implemented model (and our copy), written frequencies are used for these values.

\section{Phoneme Level}

The phoneme layer consists of 8 sets of 44 units mirroring those in the GPC level. The behavior of these units is governed by Equations 1 and 2. Excitation comes directly from the GPC system according to the GPC strength parameter, and also from consistent phonological word units by the phonology-phoneme excitation parameter. Inconsistent phonological word units and phoneme units in the same position give inhibition according to phonology-phoneme inhibition and phoneme-phoneme inhibition parameters. Output occurs when there is a complete contiguous sequence of phonemes of some length from the leftmost position in which all the phonemes exceed the stopping criterion parameter, and the rightmost phoneme of the sequence is the absent phoneme. If in any position there is more than one phoneme exceeding the criterion, the most active is taken. 


\section{Optimizing the DRC}

The target data were the correct mean response times for the 2,713 words ${ }^{3}$ in the Spieler and Balota (1997) data set that were in the model's vocabulary and were not heterophonic homographs. Assessment of fit was by $R^{2}$ for predicting these response times linearly from the number of cycles in the model and the initial phoneme (dummy) coded as a factor; we permitted the initial phoneme to be outside the scope of the model because some of the variability attributable to it will correspond to a known non-psychological mechanism (differential sensitivity of the voice key to different phonemes) ${ }^{4}$. We maximize $R^{2}$ because it is equivalent to maximizing likelihood under the assumptions of ordinary linear regression, which we use here; certain approximations do not hold if likelihood is not maximized. A case could readily be made for making different assumptions, and would definitely be necessary if the model specified the distribution of response times; this would require more sophisticated regression machinery for the type of analysis we describe and would constitute a useful generalization of our suggestion here. A heavy penalty was applied to wrong responses so that the optimization procedure would discount parameter sets that did not produce perfect accuracy.

The optimization was based on the version of the Nelder-Mead simplex procedure in the GNU R statistical suite, which interfaced with our C++-programmed DRC routine to obtain response times for given parameter sets. Our routine gives the same response time and pronunciation outputs as the original and the same results as the Coltheart et al. (2001) paper (except that the original did not implement the decay parameter). At each stage the currently best parameters (or at the beginning, the standard parameters of Coltheart et al. (2001)) were used as starting values in one run, and a further 8-12 (depending on availability of processing time) were run in parallel using starting values that were chosen by adding random noise to the best set. The simplex procedure is a standard non-gradient local search method based on examining the corners of a region of 
interest, reflecting to adjacent regions to find improved objective values, expanding the region in directions of high improvment, and shrinking the region when movement fails to yield improvement. The addition of noise was designed to counteract the local properties of this otherwise robust procedure. The stage was terminated for each run of the simplex procedure when 500 evaluations of the model had been completed (the default in R, again to counteract the effects of local tendencies in the procedure) or if it was sooner, a week had passed (an external constraint on our computing time).

The processors used for this program were $2.4 \mathrm{GHz}$ Pentium 4 Xeons, able to complete a single run of the model over all words in ca. 20 minutes (depending on the total number of cycles used for the parameters in question; this coincidentally meant that one week and 500 runs were approximately the same duration). After approximately six months, neither the simplex procedure nor adding random noise to the parameters resulted in noticeable improvement in the fit of the model; the best parameters at that point were taken as the overall best parameters for the data set. Optimization for the single-route model was similar, although faster as fewer parameters were to be optimized.

\section{Results}

\section{Overall Fit}

We first examine overall performance in the manner of the regression approach. The obtained best parameters are listed in Table 1. Initial phoneme accounted for $37.04 \%$ of the variance. The DRC with standard parameters plus initial phoneme accounted for $43.09 \%$ of the variance. The DRC with best parameters plus initial phoneme accounted for $50.10 \%$ of the variance in the response times. The corresponding best SRC fit accounted for less variance: $48.56 \%$ of the total. Thus the use of optimal parameters is clearly essential; it is noteworthy that even the SRC with optimal parameters accounts for more variance than the DRC with the standard parameters. To aid comparison, the 
standard adjustment to $R^{2}$ for overfitting per additional linear parameter would be 0.02-0.03\%, and the DRC has only three more parameters than the SRC, suggesting that a nonlexical mechanism is necessary in the model; it will be seen that it indeed allows a better (but imperfect) account of regularity effects.

By way of comparison, the standard predictors of letter length, log CELEX frequency ${ }^{5}$ and orthographic $N$ along with initial phoneme accounted for $51.10 \%$ of the variance. The regression model has many fewer parameters than the DRC, but accounts for more of the variance. Moreover, this regression model gives an underestimate of the variance the DRC ought to be able to account for because it is also supposed to explain the regularity effect, the position of irregularity effect, the interaction between frequency and regularity, and there is also evidence of an interaction between orthographic $N$ and frequency, but these factors are not included in the regression. This illustrates the key weakness of the factorial approach: Even though the DRC should account for the effects in the regression and more according to the results of factorial analyses, it falls short when these effects are assessed on an item-by-item basis.

\section{Relative Predictor Strength}

Before turning to a more complete set of predictors (including those considered by Coltheart et al., 2001, such as the frequency by regularity interaction, and more), we first ask where the DRC model falls down with these predictors. We examined regression models with these predictors taking the data, the models, and the models' error in predicting the data ${ }^{6}$ as the response (after onset had been covaried out). In Table 2 , it can be seen that although the model does predict a robust ( $\sim 5$ s.e. from zero) effect of orthographic $N$, the predicted effect is noticeably ( $\sim 7$ s.e.) weaker than that in the data. To reiterate, the model predicts the qualitative effects, but it does not correspond to the data when simultaneously evaluated on these effects. Neighborhood effects will be 
examined in more detail below.

\section{Effects in Word Naming 1: Core Effects}

We first examine the core effects that Coltheart et al. (2001) claim to account for with the DRC: (i) frequency, (ii) regularity and the frequency by regularity interaction, (iii) position of (first) irregularity, (iv) rime consistency, and (v) length.

\section{Frequency Effect.}

To examine the predictions for word frequency of the model, we used analyses in which several other effects were partialed out, viz.: Length, orthographic $N$, regularity, position of (first) irregularity, and first phoneme. The frequency effect is illustrated in Figure 1 for the DRC and SRC. It appears that both models' predictions follow log frequency quite closely, possibly with a slight concavity up on the log frequency scale. There is also upwards concavity in the human naming data, largely contributed by frequency saturation (e.g., Gordon \& Caramazza, 1985) such that there is little effect of frequency at high frequencies (above ca. 2000 or more on raw CELEX count). This second order curvature has been assessed by Balota et al. (2004) with regressions using quadratic $\log$ frequency. However, there is some indication in the figure of runs of misprediction in the midrange (underprediction just below a frequency of 100, and overprediction just above) that may reflect higher order components of curvature in the data.

We therefore examined models with linear, quadratic and cubic trends in log frequency, with the results in Table 3. There was evidence in the data of a positive cubic trend modifying a negative trend in the linear and quadratic contrasts. Errors in the DRC's predictions appeared to be systematically related to frequency: Its predictions were dominated by the linear negative trend, and the errors had a systematic relationship to a cubic polynomial in log frequency (as a block, $p<.05$ ). Errors in the SRC predictions were also systematically linked to such a function, although its cubic trend is in the correct 
direction $(p<.05$ on the omnibus test). One possible reason for the discrepancy is that frequency saturation results from frequency not being the relevant factor. A candidate alternative factor is contextual diversity, the number of contexts (operationalized as corpus documents) in which a word appears. This factor is inherently highly correlated with frequency, and there is evidence that it might account for the apparent effects of frequency (Adelman, Brown, \& Quesada, 2006; see also McDonald \& Shillcock, 2001, Adelman \& Brown, in press). High frequency items are likely to be needed relatively unpredictably in practically any context, and so their accessibility effectively needs to be at ceiling.

\section{Regularity and Frequency.}

A key qualification on the frequency effect comes, however, from its interaction with regularity: Words whose pronunciation is unusual given their spelling (exceptions) are named slower than those whose pronunciation is usual (regulars), but this effect is diminished if the words are of high frequency (e.g., Seidenberg, Waters, Barnes, \& Tanenhaus, 1984; Taraban \& McClelland, 1987, but see also Jared, 2002). This interaction is viewed as very important in validating models of word naming (e.g., Seidenberg, 1985; Norris \& Brown, 1986; Seidenberg, 1986; Paap, McDonald, Schvaneveldt, \& Noel, 1987; Seidenberg \& McClelland, 1989; Coltheart, Curtis, Atkins, \& Haller, 1993; Norris, 1994; Plaut et al., 1996; Kwantes \& Mewhort, 1999; Coltheart et al., 2001).

The relevant data and predictions for this interaction are presented in Figure 2 for the DRC, and Figure 3 for the SRC. Regularity was operationalized using the DRC's nonlexical route: A word was an exception if the pronunciation ultimately assigned by the nonlexical route differed from the vocabulary pronunciation; $444(16.4 \%)$ of the words were identified as exceptions. Although Glushko (1979) suggested that a model of the SRC type might support spelling-sound inconsistency effects, in terms of the dual route explanation, it is not surprising that the SRC was relatively immune to the regularity effect; although the lexical route supports some form of analogy processing of neighbors, 
this processing does not generate a full exception effect. The DRC exhibited a clear effect of regularity, with a slight apparent interaction with frequency, as the exception penalty was higher at the lowest frequencies. This occurs in the model because exception words must typically wait until the irregular phoneme is sufficiently activated by the lexical route, and this occurs more slowly for the lower frequency words as they have a stronger negative bias on their net input. The data were more noisy, but there seems to be evidence of frequency saturation for both exception and regular words with both having response times in the region of $465 \mathrm{~ms}$ for frequencies in excess of 2000.

To quantify the frequency by regularity interaction, we fitted a main exception effect and distinct cubic functions of log frequency for regulars and exceptions to data and models after the covariates listed for frequency (excepting position of irregularity). Table 4 confirms that the SRC failed to predict an exception effect. Although both the DRC and SRC predicted the strong linear component of log frequency for exception words, and the individual components of the cubic polynomial in log frequency did not reach significance in the regression with model errors, overall these errors were predicted by the complete cubic in $\log$ frequency $(p s<.01)$. Further, the DRC fitted the frequency effect for regular words poorly $(p<.001)$, but the SRC showed no evidence of misfitting this effect $(p>.1)$. Thus, although the frequency by regularity interaction could be captured qualitatively by the DRC, this did not correspond to correct prediction at a more detailed level.

\section{Position of (First) Irregularity.}

A line of evidence adduced in favor of a dual-route model with a serial nonlexical process is the influence of the position of irregularity on the exception effect (Coltheart \& Rastle, 1994; Rastle \& Coltheart, 1999; Roberts, Rastle, Coltheart, \& Besner, 2003; but see also Zorzi, 2000; Rastle \& Coltheart, 2000). It appears that exception costs for items whose first irregularity occurs in the production of earlier phonemes are greater than those for items whose irregularity is later. The DRC model predicts a position of irregularity 
effect because the nonlexical route's output can compete more strongly for earlier phonemes because they are generated sooner by the letters toward the left of the word.

Exception costs for the naming data and the DRC (covarying first phoneme, letter length, orthographic $N$, and full cubic log frequency by regularity interaction) are described in Table 5 (because there were on average no exception costs for the SRC, it was omitted from the analyses). The position of irregularity is given by the first phoneme at which the nonlexical and vocabulary pronunciations differ.

As in Rastle and Coltheart's (1999) data, there was a large exception cost for the first position of irregularity, and a smaller cost for second position, but the third position cost is non-zero and quite similar to the second position cost. This last point contrasts with the lack of a significant third position effect in the data of Rastle and Coltheart and Roberts et al. (2003). It is possible that one or more of the results is an error - there could be insufficient power in the small experiments, or a type I error in the mega-study — or it might be the case that the choice of items or participants systematically affects the results, and a model would be correct to be able to predict both patterns with differing parameters. In light of the latter possibility, we refrain from discounting either pattern of results on the basis of the current data. In the DRC, the effect of irregularity decreases with position, but the best parameter fit of the DRC underpredicts the cost at Position 1 for the Spieler and Balota data, and overpredicts it at Position 2.

Given that the form (or at least, the strength) of the frequency effect depends on the regularity of the stimulus, and there are effects of the position of irregularity over and above the effect of its presence, it seems plausible that there might be different frequency effects at different positions of irregularity. There was no evidence of this for the data $(p>.2)$, and although the DRC did predict some interaction $(p<.05)$, the interaction did not predict the model's error $(p>.1)$, suggesting that the apparent difference was an artefact of the smaller standard error of model predicted response times. 


\section{Rime Consistency.}

We sought a main effect of rime consistency (Glushko, 1979), the ratio of friends (words that look as though they should rhyme, and do) to friends plus enemies (words that look as though they should rhyme, but do not), after the previously discussed variables were covaried out, using types only, frequency weighted types (i.e., tokens), and log-frequency weighted types for the ratios in three separate analyses,${ }^{7}$ presented in Table 6. All three showed a significant effect in the data. Further analyses showed that either the types ratio or the log-weighted ratio could subsume the effect of the tokens ratio. The types and log-weighted ratios were mutually redundant, so only the former was retained as a covariate (it had a very slightly higher $\Delta R^{2}$ ). The DRC predicted a much weaker effect than that in the data, and the SRC none at all.

\section{(Letter) Length.}

As has been seen (in Table 2), there is an effect of number of letters on naming latency, each letter costing ca. $4 \mathrm{~ms}$, and this is more-or-less accurately predicted by both single- and dual-route models. This remains the case with additional covariates.

\section{Effects in Word Naming 2: Peripheral Effects}

We now move beyond Coltheart et al.'s (2001) main list of effects to examine: (i) neighborhood size (although they did examine this variable, see above); (ii) number of irregularities; (iii) the length by regularity interaction; (iv) whammy effects; (v) the influence of whammying on regularity effects; (vi) number of phonemes; (vii) body neighborhood size; and (viii) interactions of frequency with consistency variables.

\section{Neighborhood Size (Revisited).}

The analyses above indicated that the orthographic $N$ effect was predicted to be weaker in the DRC than was observed in the data. According to Coltheart et al. (2001), facilitatory neighborhood effects occur in the DRC model because the orthographically 
similar neighbors' phonological representations are activated and in turn activate their constituent phonemes, most of which will be consistent with the correct pronunciation. The effect in the model is therefore due to lexical print-sound conversion, as confirmed by lesion studies of the model (Reynolds \& Besner, 2002).

If the orthographic $N$ effect is indeed due to lexical print-sound conversion, then those orthographic neighbors that are also phonological neighbors (i.e. differ in exactly one phoneme) should have a strong facilitatory influence in both the model and data. Peereman and Content (1997) termed these phonographic neighbors, and they have found evidence that it is specifically these phonographic neighbors, rather than all the orthographic neighbors, that drive orthographic neighborhood effects for French words and pseudowords (Peereman \& Content, 1995, 1997). Moreover, Adelman and Brown (2007) have found evidence of the same effect from four mega-studies in English (including the one on which these analyses are based). Mulatti, Reynolds, and Besner (2006) have argued in contrast that the (whole) phonological neighborhood drives neighborhood effects rather than specifically the phonographic neighbors.

Table 7 shows analyses seeking simultaneous effects of the three neighborhood size variables. Only phonographic neighborhood size had a significant effect in the data. For both the DRC and SRC, an underestimation of the phonographic neighborhood effect was significant, as was an overestimation of facilitation from the phonological neighborhood for the SRC. For further analyses, we removed both the orthographic and phonological neighborhood sizes as both remained non-significant after the other was removed.

It was also the case that the introduction of phonographic $N$ (in place of orthographic $N$ ) almost halved the estimated effect of the rime consistency ratio to a marginal -2.697 (SE 1.600, $p=.092)$. We did not eliminate it as a covariate at this point because once the next two variables (number of irregularities and the regularity by length interaction) were partialed out, the estimate increased to a significant -3.551 ( $S E$ 1.606). 


\section{Number of Irregularities.}

Since irregularities in pronunciation relative to grapheme-phoneme correspondences produce slower responses, it seems reasonable to ask whether increasing numbers of irregularities further slow responses. This would not be expected if exception costs are due to the erasure of fast assembled phonology as in the dual route model described by Norris and Brown (1986), but would be expected if exception effects occur due to the serial resolution of phonological competition as in LEX (Kwantes \& Mewhort, 1999).

Here, as there are few monosyllables with more than two irregularities, we simply divided the words into those with a single irregularity and those with many irregularities (i.e. whose correct pronunciation differed in more than one phonemic slot from those produced by the DRC rules), and sought an additional response time cost for the latter. The data showed a clear $6.879 \mathrm{~ms}(S E 1.993)$ cost, but the DRC predicted an advantage: $-1.373 \mathrm{~ms}(S E$ 0.362), which clearly differs from the effect in the data. This appears to reflect some lexical factor to which the model is sensitive, as the SRC also predicted the advantage (-1.396 ms, SE 0.497), but clearly neither model predicts the observed cost.

\section{Regularity by (Letter) Length Interaction.}

In both stage and cascade models, when two factors effect changes in speed of processing in the same component, the result is typically an interaction of the effects (Sternberg, 1969; McClelland, 1979). Adelman (2005) examined length and regularity effects, which both relate to the nonlexical component of the DRC, and observed that the DRC with standard parameters showed an subadditive interaction of regularity and length.

We examined which, if any, interaction between these variables obtained in the Spieler and Balota (1997) data (after partialing out regularity by cubic log frequency, position of irregularity, plurality of irregularity, phonographic $N$ and rime consistency ratio). The interaction was superadditive $(p<.01)$, with a $6.978 \mathrm{~ms} /$ letter $(S E 0.991)$ effect for exception words (which are already slower) and a $3.700 \mathrm{~ms} /$ letter (SE 0.486) 
effect for regulars. By contrast, the DRC showed a $5.562 \mathrm{~ms} /$ letter ( $S E$ 0.070) effect for regulars, but a $-0.479 \mathrm{~ms} /$ letter ( $S E$ 0.143) effect for exceptions. Clearly, the interaction predicted model error $(p<.001)$. The SRC showed an superadditive interaction $(p<.01)$, $4.429 \mathrm{~ms} /$ letter $(S E 0.247)$ for exceptions and $3.707 \mathrm{~ms} /$ letter $(S E 0.121)$ for regulars, but its size was smaller than that in the data $(p<.05)$.

\section{Position of (First) Whammy.}

Rastle and Coltheart (1998) introduced the term whammy to describe the situation where the spelling-sound correspondence for a digraph (a two-letter grapheme) differs from the regular pronunciation of the digraph's first letter. For instance, SH is regularly pronounced / $S$ / (in the notation of the model) as in SHIP, but $\mathrm{S}$ without a following $\mathrm{H}$ is regularly pronounced /s/ as in SIP. In processing these words, the DRC's nonlexical route pronunciation will be temporarily erroneous (for regulars) when only first letter of the digraph is available. The model might therefore predict a slowing for these whammied words, whose temporarily incorrectly parsed. Rastle and Coltheart confirmed for a set of pronounceable pseudowords with and without whammies that both people and the model produced slower responses for those that were whammied.

Of course, neither the model nor human participants need show the same results in response to words as to nonwords, and the model's best parameters for these data are different from those in the simulations of Rastle and Coltheart (1998). Table 8 describes the relevant analyses. We considered a word whammied only if the pronunciation of the whole word was regular and a phoneme was temporarily mistranslated by the grapheme-phoneme route due to letters being unavailable. The data showed a response time cost for first position whammies and a benefit for second position whammies. Although the DRC made predictions in the correct direction for these positions, they were much smaller than in the data. The fifth position whammying effect that is significant in the model predictions is larger, but does not reach significance, in the data. The SRC 
predictions bear little resemblance to the data.

Influences of Digraphy (Whammying) on Regularity Effects.

Andrews, Woollams, and Bond (2005) investigated the suggestion of Coltheart et al. (2001) that apparent rime-consistency effects might be due to whammying, as a confound between whammying and consistency appeared to underly the ability of the DRC model to predict the condition means of an experiment of Jared (1997) with a consistency manipulation. In two experiments, Andrews et al. factorially manipulated spelling-sound regularity and consistency with the presence of digraphs. They found that both irregular words and inconsistent words were only slower than regular control words when the irregularity or inconsistency arose from a digraph that would have been whammied were the word regular. We divided the irregular words in the Spieler and Balota (1997) data into those that met this criterion and those that did not. Replacing the standard DRC regularity criterion to one that admitted only these exception words as exceptions in the regression equation (including the interactions) changed its $R^{2}$ from $55.93 \%$ to $55.89 \%$. Instead, creating a three-level regularity factor with regular, whammy-exception and nonwhammy-exception as levels, and including the interactions of this factor with cubic $\log$ frequency, position of irregularity, length and multiple irregularity, signficantly increased the $R^{2}$ to $56.39 \%(p<.01)$. We then sought to reduce the complexity of the model: The interaction with length and the interaction with cubic log frequency could be reduced to have only two levels, regular and exception; and multiple irregularities affected only exception words whose first irregularity was digraphic. This model had an $R^{2}$ of $56.31 \%(p>.5$ for the decrement).

Table 9 shows the various exception and whammying costs found in such an analysis; the SRC is omitted as it has already been seen that its predictions for whammies and exceptions are highly inconsistent with the data. It is again clear that despite being provided with the best parameters, the DRC did not predict an appropriate whammy 
effect, predicted an insufficient exception cost for first position irregularities and too great an exception cost for later irregularities, and did not predict the large effect of a second irregularity. Moreover, it did not predict the interaction between whammying and irregularity and position of irregularity; on the whole, it predicts greater exception costs for exceptions that would not be whammied, and this is the case for Position 1 irregularities (which were not examined by Andrews et al., 2004) in the data, but not Position 3 irregularities, where the opposite effect is found.

\section{Phonemic Length.}

A consequence of the manner in which the nonlexical route of the DRC generates length and whammy effects suggests that the length effects predicted by the model arise from the number of letters not the number of phonemes, and that number of phonemes should produce a facilitatory effect only due to digraphy. Therefore, we investigated whether there were phonemic length effects after the effects of the variables that have already been described. Indeed, there was a $-2.039 \mathrm{~ms} /$ phoneme ( $S E$ 0.516) effect $(p<.001)$, which the DRC predicted to be much smaller $(p<.001)$ at $-0.191 \mathrm{~ms} /$ phoneme $(S E 0.075, p<.05)$, as the effect can only arise from digraphs that are not whammies (e.g., where LL $\rightarrow / l /$ in HILL does not whammy $\mathrm{L} \rightarrow / l /$ ). The SRC predictions were in the opposite direction $(0.896, S E 0.127, p<.001)$. We will go on to discuss how this result contrains possible rules for the initiation of pronunciation in reading aloud.

Since the letter length variable's influence varied with regularity, we investigated whether a similar interaction was also observed with phonemic length, and indeed it was $(p<.05)$. The effect was $-1.500 \mathrm{~ms} /$ phoneme $(S E 0.558, p<.01)$ for regulars, but -4.930 $\mathrm{ms} /$ phoneme $(S E 1.254, p<.001)$ effect for exceptions. The DRC predicted a -0.236 $\mathrm{ms} /$ phoneme $(S E 0.081, p<.01)$ effect for regulars, but no effect for exceptions $(0.050$, $S E 0.182, p>.5)$, and the interaction significantly predicted model error $(p<.01)$.

Partialing out phonemic length removes the remaining effect of rime consistency 
$(-2.413, S E 1.646, p>.1)$, suggesting with other results that the influence of rime consistency is due to confounds with phonemic length and phonographic $N$. Rime consistency was therefore removed from the list of covariates. Once phonemic length was partialed, a small facilitation effect from phonological $N(-0.085, S E$ 0.036) reached signficance, without altering other effects. The DRC predicted the phonological $N$ effect should be inhibitory $(0.021, S E$ 0.005). The SRC correctly predicted facilitation, and the partialing out of this variable made its phonemic length prediction facilitatory. Partialing out phonological $N$ did not substantially alter the models' letter length predictions.

\section{$\operatorname{Body} N$.}

Some authors (e.g., Ziegler, Perry, Jacobs, \& Braun, 2001) have investigated body $N$, the sum of friends and enemies, as a determiner of naming latencies ${ }^{8}$. As with rime consistency, we investigated this variable in separate analyses using types only, frequency weighted types (i.e., tokens) and log frequency weighted versions of the body $N$ variable. None of these had a significant effect $(p>.1)$ after the already discussed variables had been covaried out. The types only and log-frequency weighted body $N$ showed no effect for the DRC model $(p>.1)$, and all three gave significant facilitation for the SRC $(p<.05)$.

\section{Interactions with Frequency: N, Rime Consistency and Regularity (Revisited).}

Several variables have been said to produce the regularity by frequency interaction that has been entered into the analyses so far: As well as being interpreted as a genuine interaction between regularity and frequency (e.g., Coltheart et al., 2001), it has been attributed to an interaction between consistency and frequency (e.g., Plaut et al., 1996, but see Jared, 1997). Also, as we have noted, phonographic $N$ accounts for some spelling-sound consistency effects, and Balota et al. (2004) have found an interaction between orthographic $N$ and frequency. We therefore conducted analyses with all three such interactions. As before, we used cubic log frequency, and conducted analyses that 
included the multiplicative interactions of this frequency block with each of the three types of rime consistency ratio. Only in the analysis using the token ratio did the frequency interaction reach significance, so we further consider only this ratio.

All three interactions showed significant unique effects. The phonographic $N$ by frequency interaction $(p<.001)$ was such that the advantage for words with high phonographic $N$ was smaller for high frequency words. The token rime consistency ratio by frequency interaction $(p<.05)$ was such that high consistency words showed greater frequency effects. Finally, the regularity by frequency interaction remained as expected $(p<.001)$ : Frequency effects were greater for exceptions than for regulars.

What Do These Variables Explain?

The $R^{2}$ s for the final regression models were $57.41 \%$ for the data, $98.21 \%$ for the prediction of the DRC (including onsets), $12.57 \%$ for the error in the DRC, $95.20 \%$ for the prediction of the SRC (onsets included), and $14.09 \%$ for the prediction of the SRC's error.

\section{Discussion}

There are significant difficulties in obtaining strong conclusions regarding the adequacy of models from previous effect-based factorial approaches and regression approaches based on total fit, especially when the parameters used in generating model predictions are not optimal. We argued that several difficulties can be overcome by finding optimal parameters for large data sets and performing regression analyses on model predictions for these data and the error in these predictions, in addition to regressions on the data themselves. We demonstrated the techniques with Coltheart et al.'s (2001) DRC model. One set of differences between the model and data was related to frequency effects, and we suggested that these might relate to the functional form of the bias on the lexical nodes and the possiblity that contextual diversity rather than frequency might be the causative factor (Adelman et al., 2006). Other aspects of model error appeared to be 
related to spelling-sound conversion, including the regularity by length interaction, and the multiple irregularities effect. In the following, we will explicate the manner in which the left-to-right grapheme-phoneme conversion route, a core theoretical component of the DRC, causes problems in accounting for these more subtle aspects of the spelling-sound phenomena that it is designed to explained. We will then turn our focus to the more general applicability of the method we have employed.

\section{Exception Effects and the Nonlexical Route of the DRC}

Why should the DRC's nonlexical route produce length and exception effects, but a subadditive interaction between them? To answer this question, we examined phoneme activation in DRC simulations with the words SPAT, SPATS and SPATE removed from its lexicon, and one of these replaced with either its usual regular or an altered exceptional pronunciation. Comparing SPAT and SPATS indicates the source of the length effect, and comparing regular and exceptional pronunciations illuminates the exception effect.

First, Figure 4a illustrates the activation of phonemes for reading the word SPAT when it was reinserted in the lexicon with its usual regular pronunciation $(/ \operatorname{sp}\{t /)$ and a frequency of 50. The phonemes' activation increases more quickly if they are earlier in the pronunciation since they are produced by letters further left in the input and therefore receive excitation from the nonlexical route sooner. Therefore, the last phoneme to exceed criterion is the blank phoneme (dotted line) indicating the end of the pronunciation in Position 5, which is supported by the blank letter in Position 5 of the input via the nonlexical route. The model thus produced the correct pronunciation after 85 cycles.

The source of length effects is illuminated by comparison of Figures 4a and 4b. Figure $4 \mathrm{~b}$ uses the word SPATS instead of SPAT, also with regular pronunciation (/sp $\{t s /$. Since this word is longer, the sixth phoneme is the blank that is needed to complete the pronunciation, and this receives activation from the nonlexical route when the sixth 
(blank) letter in the input is processed. This phoneme is activated more slowly than the Position 5 phoneme that is the last to exceed the stopping criterion in Figure 4a. In fact, the Position 6 blank phoneme in SPATS exceeds the stopping criterion on Cycle 92, and this is when the pronunciation is output by the model.

The comparison between Figures $4 \mathrm{a}$ and $4 \mathrm{c}$ illustrates the source of the exception effect. Figure 4c again uses the orthographic form SPAT, but with a pronunciation in the lexicon that is irregular in the first phoneme $(/ S p\{t /)$. The consequence of this is that the correct first phoneme $(/ S /$, solid line) never receives activation from the nonlexical route and receives inhibition from the now incorrect regular phoneme $(/ s /)$. Notably, although it is the conflict between the two routes' pronunciations that is intended to produce the exception effect, the vast majority of the difference between the regular and exception cases is contributed by the lack of nonlexical support, not the inhibition from the regular phoneme. The correct phoneme is therefore more slowly activated than the blank fifth phoneme, and does not reach the stopping criterion until the 100th cycle, when the model produces the new correct pronunciation. Thus the exception effect occurs due to the delaying of the irregular phoneme to be later than the final blank phoneme.

Figure $4 \mathrm{~d}$ now combines the longer orthographic form SPATs with an irregular pronunciation in the first phoneme. Again, there is a delay in the activation of the first phoneme. The final blank phoneme is now produced by the sixth letter not the fifth compared to Figure 4c. However, although the final blank phoneme reaches the stopping criterion later, it does not reach criterion later than the irregular first phoneme, which continues to reach criterion at Cycle 100, which is when the pronunciation is generated by the model. Therefore, in the presence of a strong exception slowing, there is no further slowing due to length. If there is any exception to this, it will be because the last blank phoneme is generated so late that the exceptional phoneme has no influence on response time; that is, for longer words, there may be a length effect due to the nonlexical route, 
but only in the absence of an exception effect due to the nonlexical route.

Figures $4 \mathrm{e}$ and $4 \mathrm{f}$ are designed to allow a similar examination of the number of irregularities effect. In these, a further third position irregularity has been introduced into the pronunciation in the lexicon. Note that in Table 5 we established that the model predicts a cost of irregularity at this position with these parameters, and it is smaller than that in earlier positions. In a similar fashion to the absent length effect, the irregularity in Phoneme 3 slows the activation of that phoneme, but less so than the irregularity in first position. Moreover, this must be the case if the position of irregularity effect is to obtain. For both of these words then, the first phoneme is again the last to reach the stopping criterion after 100 cycles, when they are pronounced.

\section{Decision Rules and Exception Effects.}

Given these incorrect effects arise in the model because of the interaction between the nonlexical route and the decision rule for readiness of pronunciation, an alteration to this decision rule might ameliorate the problem. An additive rule, comparing the sum of phoneme activations to a stopping criterion, would give an effect of multiple irregularities. However, this would probably create too large a facilitation from number of phonemes, since a four phoneme word would have approximately 1.25 times the activation of a three phoneme word, which would exceed the size of the letter length effect. An averaging (arithmetic mean) rule would have a different problem: Since later phonemes lag in activation, words with many phonemes will have lower average activation than those with few, in contradiction to the facilitatory phonemes effect.

A product of activations rule would have similar problems to a sum rule, as taking logarithms on both sides gives a stopping criterion rule that is sum of log activations exceeds log criterion. The same logic applies to the use of a geometric mean rule rather than an arithmetic mean rule. A more complex rule might better accommodate all the effects, but this could involve an explicit adjustment for number of phonemes. 


\section{Beyond the DRC}

Modifications to the DRC.

The DRC model proposed by Coltheart et al. (2001) clearly has some difficulties in accounting for the data under consideration here. However, this is not to say that there is no cascaded model with an addressed-assembled dual-route structure that can account for the data. It could yet be demonstrated that a modification to certain assumptions would reveal a model capable of capturing the effects without changing the basic structure. This can only be determined by further simulations. Candidate modifications suggested by the evidence here involve changing the decision rule for pronunciation to accommodate multiple irregularity effects; and adding perceptibility effects to the accumulation of letter information, possibly at the expense of seriality in the nonlexical route to better model position of irregularity. A modification suggested in part by evidence described elsewhere (Adelman et al., 2006; Adelman \& Brown, in press) is to replace frequency with contextual diversity in the bias on word nodes.

\section{Models other than the DRC.}

There are of course other models that could be assessed in a fashion similar to that used in the analysis of the DRC here. One candidate is the recent extension to the connectionist dual-process model (Perry, Ziegler, \& Zorzi, 2007) partially based on the DRC; this model was published well after the optimizations described in this paper were begun. Given that we have identified the stopping rule as a key component to be changed in the DRC, the CDP+'s alternative mechanism will be of interest.

Parallel-distributed processing models (e.g., Plaut et al., 1996) might also be assessed with optimization and model error regressions. There are a few ways, however, in which such an analysis would be more difficult or less satisfactory than the one given here, mostly centering on optimization. First, these models typically have more connections 
than the DRC and a training period, which make them slower to run. Second, most such models have a random component, either in the initial weights, or the order of word presentation (e.g., Harm \& Seidenberg, 1999); it would therefore be necessary to have several runs of the model to determine an average performance, or to treat these as parameters themselves (cf. Zevin \& Seidenberg, 2006). Third, the choice of distribution for such random components would likely influence the results, even though such distributions are not obviously parameters of the models. Fourth, some of the parameters that might be optimized - number of hidden nodes, learning rate, momentum, etc. — map indirectly onto the mechanisms of processing for reading words. Fifth, and more generally, because the mechanism that is specified is at the level of learning, potential modifications need to be at this level. To generate psychological understanding to motivate such potential modifications will therefore require more analysis than when the on-line processing is specified more directly. Nevertheless, as computing speed allows, analyses based on the principles we have described will strongly constrain the development of these models.

Our discussion here does not exhaust all models to which the method might be applied, but no further special problems are foreseeable with any other models.

\section{Beyond these data}

Of course, the method may also be extended to other data sets. To correctly perform these analyses on other data sets, the optimal parameters issue must be once again addressed: Parameters optimized for one data set will not be optimal for another, not only because of trial-to-trial variation, but more importantly because of systematic participant, item and other design factors. Such differences are important; with the parameters given here, the pronunciation crietrion was reached by the DRC for fewer than $1 \%$ of the words from Andrews and Scarratt's (1998) experimental nonwords (i.e., no response was made at all for the others). This does not invalidate the parameters: It 
would be perfectly reasonable for one set of parameters to represent the strategy for a list of words like Spieler and Balota's (1997), and another to represent the strategy for lists containing nonwords like Andrews and Scarratt's.

\section{Caveat: Individual differences.}

The problematic effects found in our analyses here are fairly simple effects. However, there is a further, and serious, concern when fitting complex models with complex effects to data of the sort we have used. These data are averages over individuals, for each of whom the parameters presumably differ. To achieve reasonable fits to individual participants would require data of greater precision at an individual level, which could only be achieved from a study in which participants provide several observations for each word. If it were possible to evade or ameliorate problematic repetition (and repetition interaction) effects, there would be several advantages to such data. The first is simply that the data would be at the correct level of grain to match prediction and therefore would not be susceptible to averaging artefacts (cf. Heathcote, Brown, \& Mewhort, 2002). Second, it permits true and strict testing of models with lack-of-fit tests that do not rely upon knowledge of important variables, as an accurate upper bound on $R^{2}$ (or another fit measure) could be calculated from a saturated model, which would generate estimates of expected fit for restricted models with fewer parameters than items. Third, such data would permit data-led identification of important variables by identifying individual items for which there is evidence of misfit; that is, the data (with some non-model-specific intuition) could guide hypothesis-generation. The combination of the detail of data with assessment that rigorously examines effects of several models of several model families under the most favorable conditions (parameters) has strong promise for theoretical progress in word naming, and other areas where differences between words generate contrasts of interest. 


\section{References}

Adelman, J. S. (2005). Regularity and length effects in word naming: A test of the dual route cascaded model. In B. G. Bara, L. Barsalou, \& M. Bucciarelli (Eds.), Proceedings of the twenty-seventh annual conference of the Cognitive Science Society (pp. 57-61). Alpha, NJ: Sheridan Printing.

Adelman, J. S., \& Brown, G. D. A. (2007). Phonographic neighbors, not orthographic neighbors, determine word naming latencies. Psychonomic Bulletin $\&$ Review, 14, $455-459$.

Adelman, J. S., \& Brown, G. D. A. (in press). Modeling lexical decision: The form of frequency and diversity effects. Psychological Review.

Adelman, J. S., Brown, G. D. A., \& Quesada, J. F. (2006). Contextual diversity, not word frequency, determines word-naming and lexical decision times. Psychological Science, $17,814-823$.

Andrews, S. (1989). Frequency and neighborhood effects on lexical access: Activation or search? Journal of Experimental Psychology: Learning, Memory, and Cognition, 15, 802-814.

Andrews, S. (1992). Frequency and neighborhood effects on lexical access: Lexical similarity or orthographic redundancy? Journal of Experimental Psychology: Learning, Memory, and Cognition, 18, 234-254.

Andrews, S., \& Scarratt, D. R. (1998). Rule and analogy mechanisms in reading nonwords: Hough dou peapel rede gnew wirds? Journal of Experimental Psychology: Human Perception and Performance, 24, 1052-1086.

Andrews, S., Woollams, A., \& Bond, R. (2005). Spelling-sound typicality only affects words with digraphs: Further qualifications to the generality of the regularity effect on word naming. Journal of Memory and Language, 53, 567-593.

Baayen, R. H., Piepenbrock, R., \& Gulikers, L. (1995). The CELEX Lexical Database 
(Release 2) [CD-ROM]. Philadelphia: Linguistic Data Consortium, University of Pennsylvania.

Balota, D. A., Cortese, M. J., \& Pilotti, M. (1999). Item-level analysis of lexical decision: Results from a mega-study. In Abstracts of the 40th Annual Meeting of the Psychonomics Society (p. 44). Los Angeles, CA: Psychonomic Society.

Balota, D. A., Cortese, M. J., Sergent-Marshall, S. D., Spieler, D. H., \& Yap, M. J. (2004). Visual word recognition of single-syllable words. Journal of Experimental Psychology: General, 133, 283-316.

Balota, D. A., \& Spieler, D. H. (1998). The utility of item-level analyses in model evaluation: A reply to Seidenberg and Plaut. Psychological Science, 9, 238-240.

Besner, D. (1999). Basic processes in reading: Multiple routines in localist and connectionist models. Cambridge, MA: MIT Press.

Besner, D., \& Borowsky, R. (2006). Postscript: Plaut and Booth's (2006) new simulations - What have we learned? Psychological Review, 113, 194-195.

Besner, D., Twilley, L., McCann, R. S., \& Seergobin, K. (1990). On the association between connectionism and data: Are a few words necessary? Psychological Review, 97, 432-446.

Coltheart, M., Curtis, B., Atkins, P., \& Haller, M. (1993). Models of reading aloud: Dual-route and parallel-distributed-processing approaches. Psychological Review, $100,589-608$.

Coltheart, M., Davelaar, E., Jonasson, J. T., \& Besner, D. (1977). Access to the internal lexicon. In S. Dornič (Ed.), Attention and performance VI (pp. 535-555). Hillsdale, NJ: Erlbaum.

Coltheart, M., \& Rastle, K. (1994). Serial processing in reading aloud: Evidence for dual-route models of reading. Journal of Experimental Psychology: Human Perception and Performance, 20, 1197-1211. 
Coltheart, M., Rastle, K., Perry, C., Langdon, R., \& Ziegler, J. (2001). DRC: A dual route cascaded model of visual word recognition and reading aloud. Psychological Review, 108, 204-256.

Cutler, A. (1981). Making up materials is a confounded nuisance, or: Will we be able to run any psycholinguistic experiments at all in 1990? Cognition, 10, 65-70.

Frost, R., Kugler, T., Deutsch, A., \& Forster, K. I. (2006). Orthographic structure versus morphological structure: Principles of lexical organization in a given language. Journal of Experimental Psychology: Learning, Memory, and Cognition, 31, $1293-1326$.

Glushko, R. J. (1979). The organization and activation of orthographic knowledge in reading aloud. Journal of Experimental Psychology: Human Perception and Performance, 5, 674-691.

Gordon, B., \& Caramazza, A. (1985). Lexical access and frequency sensitivity: Frequency saturation and open/closed class equivalence. Cognition, 21, 95-115.

Grainger, J., \& Jacobs, A. M. (1996). Orthographic processing in visual word recognition: A multiple read-out model. Psychological Review, 103, 518-565.

Harm, M. W., \& Seidenberg, M. S. (1999). Phonology, reading acquisition, and dyslexia: Insights from connectionist models. Psychological Review, 106, 491-528.

Heathcote, A., Brown, S., \& Mewhort, D. J. K. (2002). The power law repealed: The case for an exponential law of practice. Psychonomic Bulletin \& Review, 7, 185-207.

Jared, D. (1997). Spelling-sound consistency affects the naming of high frequency words. Journal of Memory and Language, 36, 505-529.

Jared, D. (2002). Spelling-sound consistency and regularity effects in word naming. Journal of Memory and Language, 46, 723-750.

Kwantes, P., \& Mewhort, D. J. (1999). Modeling lexical decision and word naming as a retrieval process. Canadian Journal of Experimental Psychology, 53, 306-315. 
McClelland, J. L. (1979). On the time relations of mental processes: An examination of systems of processes in cascade. Psychological Review, 86, 287-330.

McClelland, J. L., \& Rumelhart, D. E. (1981). An interactive activation model of context effects in letter perception: Part 1. An account of basic findings. Psychological Review, 88, 375-407.

McDonald, S. A., \& Shillcock, R. C. (2001). Rethinking the word frequency effect: The neglected role of distributional information in lexical processing. Language and Speech, 44, 295-323.

Mulatti, C., Reynolds, M. G., \& Besner, D. (2006). Neighborhood effects in reading aloud: New findings and new challenges for computational models. Journal of Experimental Psychology: Human Perception and Performance, 32, 799-810.

Norris, D. (1994). A quantitative multiple-levels model of reading aloud. Journal of Experimental Psychology: Human Perception and Performance, 20, 1212-1232.

Norris, D., \& Brown, G. (1986). Race models and analogy theories: A dead heat? Reply to Seidenberg. Cognition, 20, 155-168.

Paap, K. R., McDonald, J. E., Schvaneveldt, R. W., \& Noel, R. W. (1987). Frequency and pronounceability in visually presented naming and lexical decision tasks. In M. Coltheart (Ed.), Attention and performance XII: The psychology of reading (pp. 221-243). Hillsdale, NJ: Erlbaum.

Peereman, R., \& Content, A. (1995). Neighborhood size effect in naming: Lexical activation or sublexical correspondences? Journal of Experimental Psychology: Learning, Memory, and Cognition, 21, 409-421.

Peereman, R., \& Content, A. (1997). Orthographic and phonological neighborhoods in naming: Not all neighbors are equally influential in orthographic space. Journal of Memory and Language, 37, 382-410.

Perry, C., Ziegler, J. C., \& Zorzi, M. (2007). Nested incremental modeling in the 
development of computational theories: The CDP + model of reading aloud. Psychological Review, 114, 273-315.

Plaut, D. C., McClelland, J. L., Seidenberg, M. S., \& Patterson, K. (1996).

Understanding normal and impaired reading: Computational principles in quasi-regular domains. Psychological Review, 103, 56-115.

Rastle, K., \& Coltheart, M. (1998). Whammies and double whammies: The effect of length on nonword reading. Psychonomic Bulletin \& Review, 5, 277-282.

Rastle, K., \& Coltheart, M. (1999). Serial and strategic effects in reading aloud. Journal of Experimental Psychology: Human Perception and Performance, 25, 482-503.

Rastle, K., \& Coltheart, M. (2000). Serial processing in reading aloud: Reply to Zorzi (2000). Journal of Experimental Psychology: Human Perception and Performance, 26, $1232-1235$.

Reynolds, M., \& Besner, D. (2002). Neighbourhood density effects in reading aloud: New insights from simulations with the DRC model. Canadian Journal of Experimental Psychology, 56, 310-318.

Reynolds, M., \& Besner, D. (2004). Neighbourhood density, word frequency, and spelling-sound regularity effects in naming: Similarities and differences between skilled readers and the dual route cascaded computational model. Canadian Journal of Experimental Psychology, 58, 13-31.

Roberts, M. A., Rastle, K., Coltheart, M., \& Besner, D. (2003). When parallel processing in visual word processing is not enough: New evidence from naming. Psychonomic Bulletin ES Review, 10, 405-414.

Rumelhart, D. E., \& Siple, P. (1974). The process of recognizing tachistoscopically presented words. Psychological Review, 81, 99-118.

Sears, C. R., Campbell, C. R., \& Lupker, S. J. (2006). Is there a neighborhood frequency effect in english? evidence from reading and lexical decision. Journal of 
Experimental Psychology: Human Perception and Performance, 32, 1040-1062.

Seidenberg, M. S. (1985). The time course of phonological code activation in two writing systems. Cognition, 19, 1-30.

Seidenberg, M. S. (1986). Constraining models of word recognition. Cognition, 20, 69-190.

Seidenberg, M. S., \& McClelland, J. L. (1989). A distributed, developmental model of word recognition and naming. Psychological Review, 96, 523-568.

Seidenberg, M. S., Waters, G. S., Barnes, M. A., \& Tanenhaus, M. K. (1984). When does irregular spelling or pronunciation influence word recognition. Journal of Verbal Learning and Verbal Behavior, 23, 383-404.

Siakaluk, P. D., Sears, C. R., \& Lupker, S. J. (2002). Orthographic neighborhood effects in lexical decision: The effects of nonword orthographic neighborhood size. Journal of Experimental Psychology: Human Perception and Performance, 28, 661-681.

Spieler, D. H., \& Balota, D. A. (1997). Bringing computational models of word naming down to the item level. Psychological Science, 8, 411-416.

Sternberg, S. (1969). The discovery of processing stages: Extensions of Donders' method. Acta Psychologica, 30, 276-315.

Taraban, R., \& McClelland, J. L. (1987). Conspiracy effects in word pronunciation. Journal of Memory and Language, 26, 608-631.

Weekes, B. S. (1997). Differential effects of number of letters on word and nonword naming latency. Quarterly Journal of Experimental Psychology, 50A, 439-456.

Zevin, J. D., \& Seidenberg, M. S. (2006). Simulating consistency effects and individual differences in nonword naming: A comparison of current models. Journal of Memory and Language, 54, 145-160.

Ziegler, J., Perry, C., Jacobs, A., \& Braun, M. (2001). Identical words are read differently in different languages. Psychological Science, 12, 379-384.

Zorzi, M. (2000). Serial processing in reading aloud: No challenge for a parallel model. 
Analyzing model error in the DRC 46

Journal of Experimental Psychology: Human Perception and Performance, 26, $847-856$. 


\begin{abstract}
Author Note
Some portions of this paper are based on work presented at the Twenty-Seventh Annual Conference of the Cognitive Science Society.

This work was supported by a Warwick Postgraduate Research Fellowship to JSA and by grants RES-000-22-1558 and RES-062-23-0545 from the Economic and Social Research Council (UK) and grant F/215/AY from the Leverhulme Trust. The computing facilities were provided by the Centre for Scientific Computing of the University of Warwick.

We thank Max Coltheart, Derek Besner and an anonymous reviewer for constructive reviews, and Maura Sabatos-DeVito for comments on the manuscript.
\end{abstract}




\section{Footnotes}

${ }^{1}$ According to Coltheart et al. (2001), this value should be based on the maximum frequency on the lexicon, but it appears that this value has been hard-coded based on some other lexicon than the one that Coltheart et al. currently use. We have followed their implementation here.

${ }^{2}$ Every other component of the model is influenced by activations of other components on the previous time tick.

${ }^{3}$ Analyses are based on 2,712 words because we typically discard "rev" on the grounds that there were no correct response times in the corresponding lexical decision data of Balota, Cortese, and Pilotti (1999), but this action was not taken until after the optimization was complete in this instance; the changes this causes to the $R^{2}$ values are of the order of $.01 \%$.

${ }^{4}$ This method of controlling acoustic components is intended to extend that used in Spieler and Balota's (1997) analyses which used the major articulatory features of the first phoneme, as it does not rely on there being no interactions between the effects of such features. As in other investigations that have regressed out aspects of the first phoneme for this purpose (to our knowledge, all investigations that have used mega-study data [where mega is taken to refer to the number of words]) this does not control for influence of subsequent phonemes.

${ }^{5}$ We use the CELEX (Baayen, Piepenbrock, \& Gulikers, 1995) written frequency because this is the source of the frequencies in the DRC model's lexicon. This makes it possible to assert that differences between the regression models and the DRC are not due to the quality of frequency count.

${ }^{6}$ Note that optimizing for a single data set fixes the intercept and slope. This may lead to a slight hypersensitivity for the detection of errors, but the effect will be small for such a large number of items in the analysis, and in any case we will concentrate on strong 
Analyzing model error in the DRC 49

effects.

${ }^{7}$ We excluded MITT from the frequency weighted analyses because the denominator of the ratio was zero.

${ }^{8}$ We thank Marco Zorzi for bringing this variable to our attention. 
Analyzing model error in the DRC 50

Table 1

Best parameters for the DRC and SRC models to fit Spieler \& Balota (1997) naming RTs.

\begin{tabular}{rrrrrr} 
Parameter & Best DRC & Best SRC & Parameter & Best DRC & Best SRC \\
\hline activation rate & .2806 & .1780 & frequency scale & .0659 & .0464 \\
stopping criterion & .3906 & .4785 & feature-letter excit. & .0051 & .0109 \\
feature-letter inhib. & -.1464 & -.1351 & letter-orthography excit. & .0752 & .0653 \\
letter-orthography inhib. & -.6361 & -.5424 & letter-letter inhib. & .0000 & .0000 \\
letter decay & .1119 & .0764 & orthography-phonology excit. & .2475 & .3307 \\
orthography-letter excit. & .3793 & .3704 & orthography-letter inhib. & .0000 & .0000 \\
orthography-orthography inhib. & -.0504 & -.0101 & orthography decay & .0039 & .0002 \\
phonology-phoneme excit. & .1344 & .1442 & phonology-phoneme inhib. & .0000 & .0000 \\
phonology-orthography excit. & .1666 & .2081 & phonology-phonology inhib. & -.0802 & -.0297 \\
phonology decay & .0027 & .0002 & phoneme-phonology excit. & .0367 & .0795 \\
phoneme-decay & .0509 & .0035 & phoneme-phoneme inhib. & -.1302 & -.1496 \\
GPC delay & 10.0000 & - & GPC strength & .0441 & - \\
intercept & 357.5082 & 359.1956 & GPC rate & 16.0000 & -
\end{tabular}


Data

DRC

DRC Error

SRC

SRC Error

$\begin{array}{rccccc}\text { Log Frequency } & -2.468(0.145) * * * & -2.382(0.040) * * * & -0.086(0.145) & -2.334(0.036) * * * & -0.134(0.147) \\ \text { Number of Letters } & 3.917(0.500) * * * & 4.707(0.137) * * * & -0.789(0.499) & 3.687(0.124) * * * & 0.230(0.506) \\ \text { Orthographic } N & -0.597(0.072) * * * & -0.101(0.020)^{* * *} & -0.496(0.072) * * * & -0.202(0.018)^{* * *} & -0.395(0.073) * * *\end{array}$

Log Frequency is the natural logarithm of (CELEX frequency plus one). Tests for comparison with zero: ${ }^{\dagger} p<.1{ }^{*} p<.05 ; * * p<.01 ; * * * p<.001$.

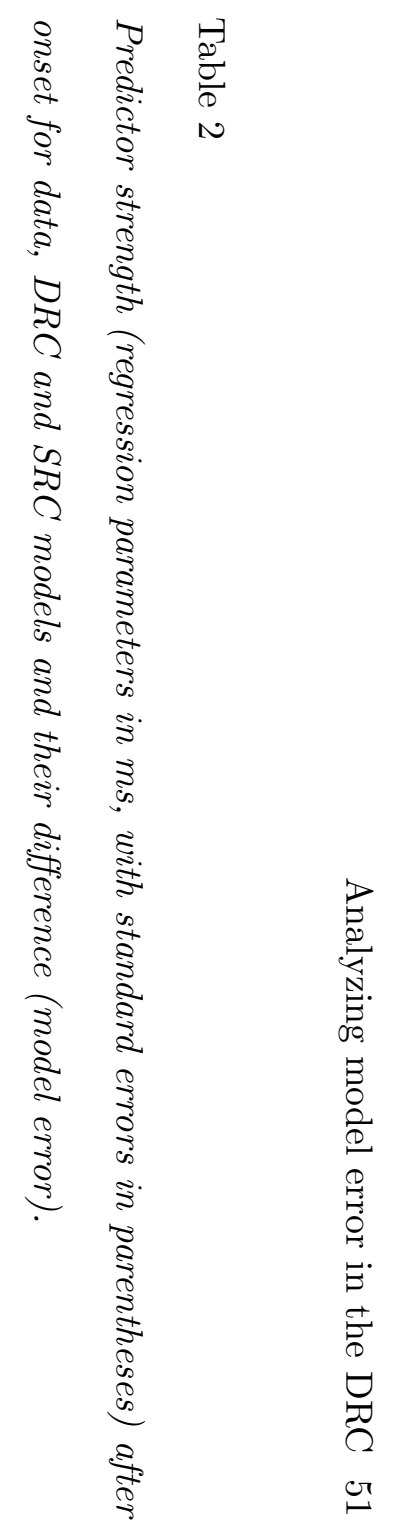




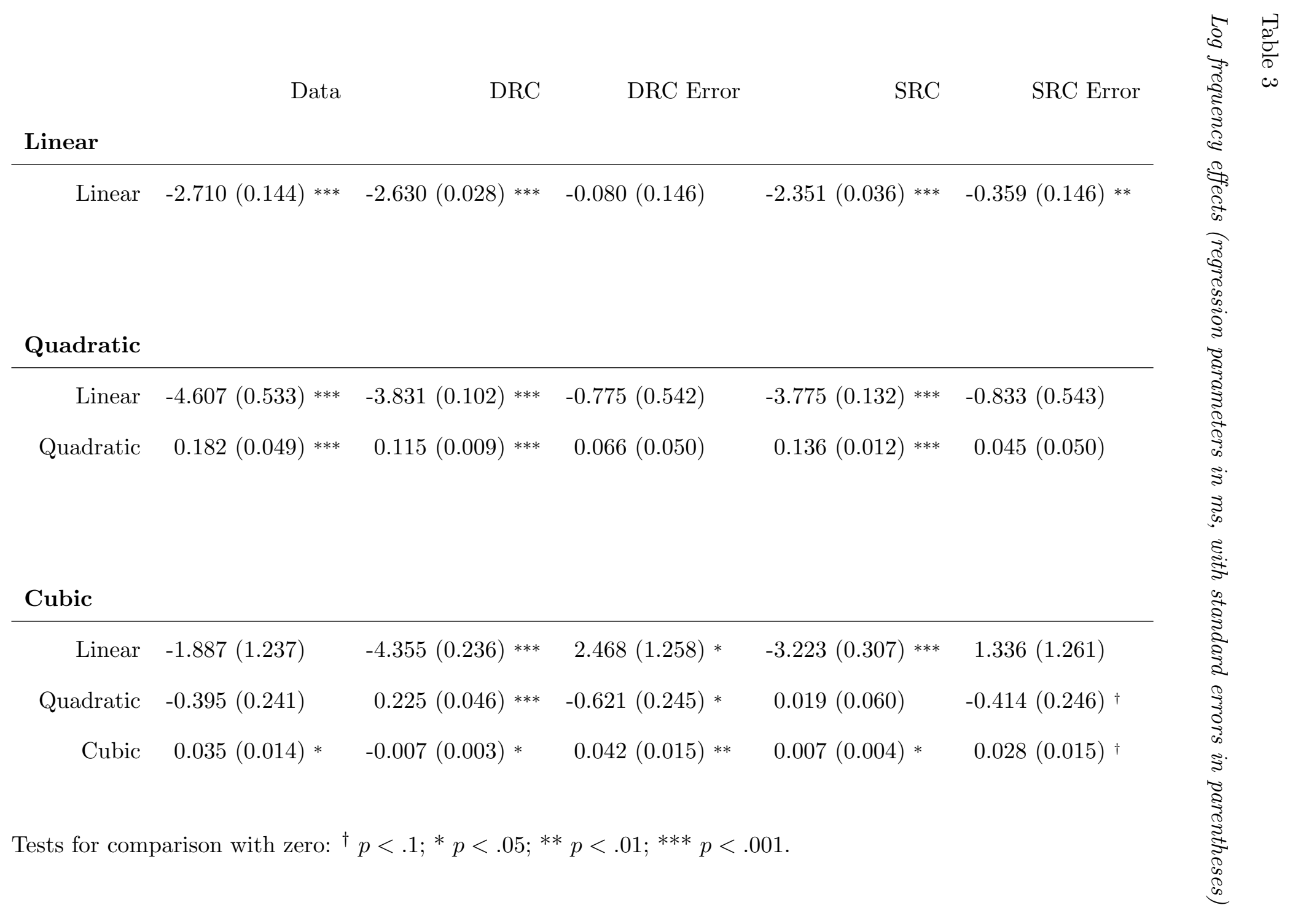




\begin{tabular}{|c|c|c|c|c|c|}
\hline & Data & $\mathrm{DRC}$ & DRC Error & $\mathrm{SRC}$ & SRC Error \\
\hline Exception costs & $8.155(0.838)^{* * *}$ & $8.137(0.167)^{* * *}$ & $0.617(0.853)$ & $0.730(0.207)^{* * *}$ & $7.425(0.855)^{* * *}$ \\
\hline \multicolumn{6}{|l|}{ Regulars } \\
\hline Linear & $-1.632(1.336)$ & $-4.670(0.267)^{* * *}$ & $3.038(1.360) *$ & $-3.220(0.330) * * *$ & $1.588(1.364)$ \\
\hline Quadratic & $-0.411(0.267)$ & $0.321(0.053) * * *$ & $-0.731(0.272) * *$ & $0.025(0.066)$ & $-0.436(0.273)$ \\
\hline Cubic & $0.038(0.016) *$ & $-0.012(0.003)^{* * *}$ & $0.050(0.017)^{* *}$ & $0.007(0.004) \dagger$ & $0.031(0.017) \dagger$ \\
\hline \multicolumn{6}{|l|}{ Exceptions } \\
\hline Linear & $-8.104(3.745) *$ & $-5.614(0.748) * * *$ & $-2.490(3.814)$ & $-4.060(0.925) * * *$ & $-4.044(3.825)$ \\
\hline Quadratic & $0.076(0.653)$ & $0.149(0.131)$ & $-0.073(0.665)$ & $0.093(0.161)$ & $-0.017(0.667)$ \\
\hline Cubic & $0.028(0.035)$ & $0.006(0.003)$ & $0.021(0.036)$ & $0.005(0.009)$ & $0.023(0.036)$ \\
\hline
\end{tabular}

Tests for comparison with zero: ${ }^{\dagger} p<.1{ }^{*} p<.05 ;{ }^{* *} p<.01 ;{ }^{* * *} p<.001$. Exceptions are identified as words that are ultimately parsed by the DRC's nonlexical route in a way that supports a phoneme that differs from the vocabulary pronunciation. 


\begin{tabular}{rrrrrr} 
Data & DRC & DRC Error & SRC & SRC Error \\
\hline 1 & $27.132(2.863) * * *$ & $13.795(0.521)^{* * *}$ & $13.338(2.927) * * *$ & $0.558(0.714)$ & $26.574(2.924) * * *$ \\
2 & $7.470(1.050)^{* * *}$ & $10.093(0.191)^{* * *}$ & $-2.623(1.073) *$ & $0.617(0.262) *$ & $6.854(1.073) * * *$ \\
3 & $6.613(1.449) * * *$ & $4.463(0.264)^{* * *}$ & $1.982(1.481)$ & $0.891(0.361) *$ & $5.723(1.480) * * *$ \\
4 & $1.259(3.053)$ & $1.631(0.556)^{* *}$ & $-0.372(3.122)$ & $1.345(0.762) \dagger$ & $-0.086(3.119)$ \\
5 & $1.761(6.626)$ & $-2.112(1.207){ }^{*}$ & $3.874(6.774)$ & $0.232(1.653)$ & $1.530(6.768)$
\end{tabular}

Exceptions are identified as words that are ultimately parsed by the DRC's nonlexical route in a way that supports

a phoneme that differs from the vocabulary pronunciation, coded by the position of that phoneme. Tests for comparison with zero: ${ }^{\dagger} p<.1 ;^{*} p<.05 ; * * p<.01 ; * * * p<.001$. 


\begin{tabular}{rrrrrr}
\multicolumn{1}{c}{ Data } & DRC & DRC Error & SRC & SRC Error \\
\hline By types & $-5.571(1.588) * * *$ & $-0.663(0.289) *$ & $-4.908(1.624) * *$ & $-0.284(0.397)$ & $-5.286(1.623) * *$ \\
\hline By tokens & $-2.322(1.091) *$ & $-0.546(0.199) * *$ & $-1.776(1.116)$ & $-0.062(0.272)$ & $-2.259(1.114) *$ \\
\hline Log-weighted & $-5.240(1.542) * * *$ & $-0.661(0.281) *$ & $-4.579(1.578)$ & $-0.231(0.385)$ & $-5.008(1.576) * *$
\end{tabular}

Each ratio is entered is a separate analysis. Tests for comparison with zero: ${ }^{\dagger} p<.1 ;{ }^{*} p<.05 ;{ }^{* *} p<.01 ;{ }^{* *} p<.001$ 


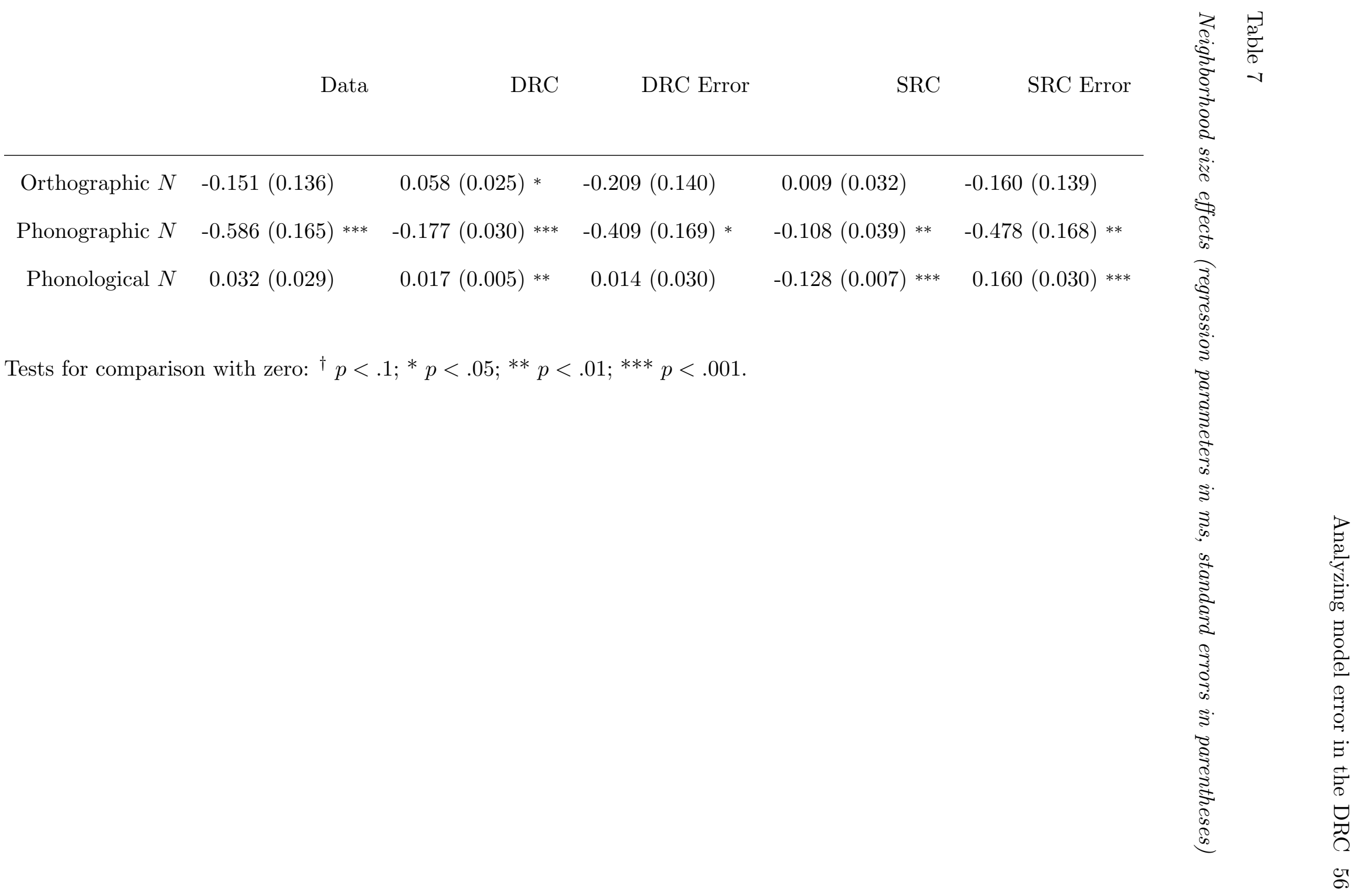




\begin{tabular}{llllll}
\multicolumn{7}{c}{ Data } & DRC & DRC Error & SRC & SRC Error \\
\hline 1 & $8.706(2.039) * * *$ & $0.268(0.294)$ & $8.438(2.065) * * *$ & $-3.133(0.502) * * *$ & $11.838(2.074) * * *$ \\
2 & $-2.486(0.987) *$ & $-0.157(0.143)$ & $-2.329(0.999) *$ & $0.219(0.243)$ & $-2.705(1.003) * *$ \\
3 & $0.491(1.088)$ & $0.206(0.157)$ & $0.284(1.101)$ & $0.096(0.268)$ & $0.395(1.106)$ \\
4 & $-0.437(1.818)$ & $-0.027(0.263)$ & $-0.411(1.841)$ & $1.412(0.448) * *$ & $-1.850(1.849)$ \\
5 & $-6.451(3.661) \dagger$ & $-2.210(0.529) * * *$ & $-4.241(3.706)$ & $6.054(0.903) * * *$ & $-12.505(3.723) * * *$
\end{tabular}

Whammies are identified in regular words that are partially parsed by the DRC's nonlexical route in a way that supports an incorrect phoneme, by the position of that phoneme. Tests for comparison with zero: $\dagger$ $p<.1{ }^{*} p<.05 ;{ }^{* *} p<.01 ; * * * p<.001$. 
Table 9

Exception and whammying costs by position (regression parameters in ms, standard errors in parentheses)

Data DRC DRC Error

\begin{tabular}{cccc}
\hline \multicolumn{3}{c}{ Whammied Regulars } \\
\hline 1 & $8.877(2.034)^{* * *}$ & $0.237(0.294)$ & $8.640(2.058) * * *$ \\
2 & $-2.480(0.983) *$ & $-0.151(0.142)$ & $-2.329(0.995) *$ \\
3 & $0.477(1.084)$ & $0.212(0.157)$ & $0.265(1.097)$ \\
4 & $-0.463(1.812)$ & $-0.017(0.262)$ & $-0.446(1.834)$ \\
5 & $-6.506(3.649)^{\dagger}$ & $-2.219(0.527))^{* * *}$ & $-4.318(3.692)$
\end{tabular}

Unwhammied Exceptions

\begin{tabular}{rrrr}
\hline 1 & $35.933(4.267)^{* * *}$ & $12.726(0.617)^{* * *}$ & $23.207(4.318)^{* * *}$ \\
2 & $2.658(1.687)$ & $10.769(0.244)^{* * *}$ & $-8.111(1.707)^{* * *}$ \\
3 & $-1.636(2.301)$ & $8.898(0.332)^{* * *}$ & $-10.534(2.328)^{* * *}$ \\
4 & $-3.538(4.123)$ & $7.701(0.596)^{* * *}$ & $-11.238(4.171)^{* *}$ \\
5 & $-11.509(14.931)$ & $13.733(2.157)^{* * *}$ & $-25.242(15.109)^{\dagger}$
\end{tabular}

Whammied Exceptions

\begin{tabular}{rccc}
\hline 1 & $20.967(3.861) * * *$ & $12.078(0.558)^{* * *}$ & $8.889(3.907) *$ \\
2 & $3.397(1.774) *$ & $9.937(0.256)^{* * *}$ & $-5.968(1.795) * * *$ \\
3 & $5.755(2.344) *$ & $8.095(0.339)^{* * *}$ & $-2.340(2.372)$ \\
4 & $-6.202(4.923)$ & $8.428(0.711)^{* * *}$ & $-14.630(4.982)^{* *}$ \\
5 & $-4.604(7.478)$ & $7.139(1.080)^{* * *}$ & $-11.743(7.567)$ \\
Multiple & $10.440(2.571) * * *$ & $0.349(0.371)$ & $10.091(2.601)^{* * *}$
\end{tabular}

Whammies are identified in words that are partially parsed by the DRC's nonlexical route in a way that temporarily supports a phoneme that differs from the regular. Exceptions are identified as words that are ultimately parsed by the DRC's nonlexical route in a way that supports a phoneme that differs from the vocabulary pronunciation. Position is determined by phoneme. Tests for comparison with zero: ${ }^{\dagger} p<.1$ * $^{*} p<.05 ;{ }^{* *} p<.01$; *** $p<.001$. 


\section{Figure Captions}

Figure 1. Effect of word frequency (covariate adjusted) on response time in DRC, SRC, and data after covariates. Error bars show $\pm 1 S E$, based on residual sum-of-squares from the model with covariates. DRC/SRC response times are based on best-fitting intercept and slope for the data set. Bands are taken on the basis of the Napierian logarithm of CELEX written frequency to the nearest third; number of items in a band is at the base of the figure.

Figure 2. Effect of word frequency by regularity on response time in DRC and data after covariates. DRC response times based on best-fitting intercept and slope for the data set. Bands are taken on the basis of the Napierian logarithm of CELEX written frequency to the nearest third. Exceptions are identified as words that are ultimately parsed by the DRC's nonlexical route in a way that supports a phoneme that differs from the vocabulary pronunciation.

Figure 3. Effect of word frequency by regularity on response time in SRC and data after covariates. SRC response times based on best-fitting intercept and slope for the data set. Bands are taken on the basis of the Napierian logarithm of CELEX written frequency to the nearest third. Exceptions are identified as words that are ultimately parsed by the DRC's nonlexical route in a way that supports a phoneme that differs from the vocabulary pronunciation.

Figure 4. Phoneme activation in the DRC with best parameters for critical phonemes over the duration of naming for 6 words inserted in the lexicon of the DRC with frequency 50 . (Spat, spats, and spate were removed from the lexicon for this exposition.) (a), (c) and (e) use the four letter orthographic form SPAT. (b), (d) and (f) use the five letter orthographic form SPATS. (a) and (b) have regular pronunciations. (c) and (d) are irregular in the first 
Analyzing model error in the DRC 60

phoneme. (e) and (f) have irregularities in both first and third phonemes. 


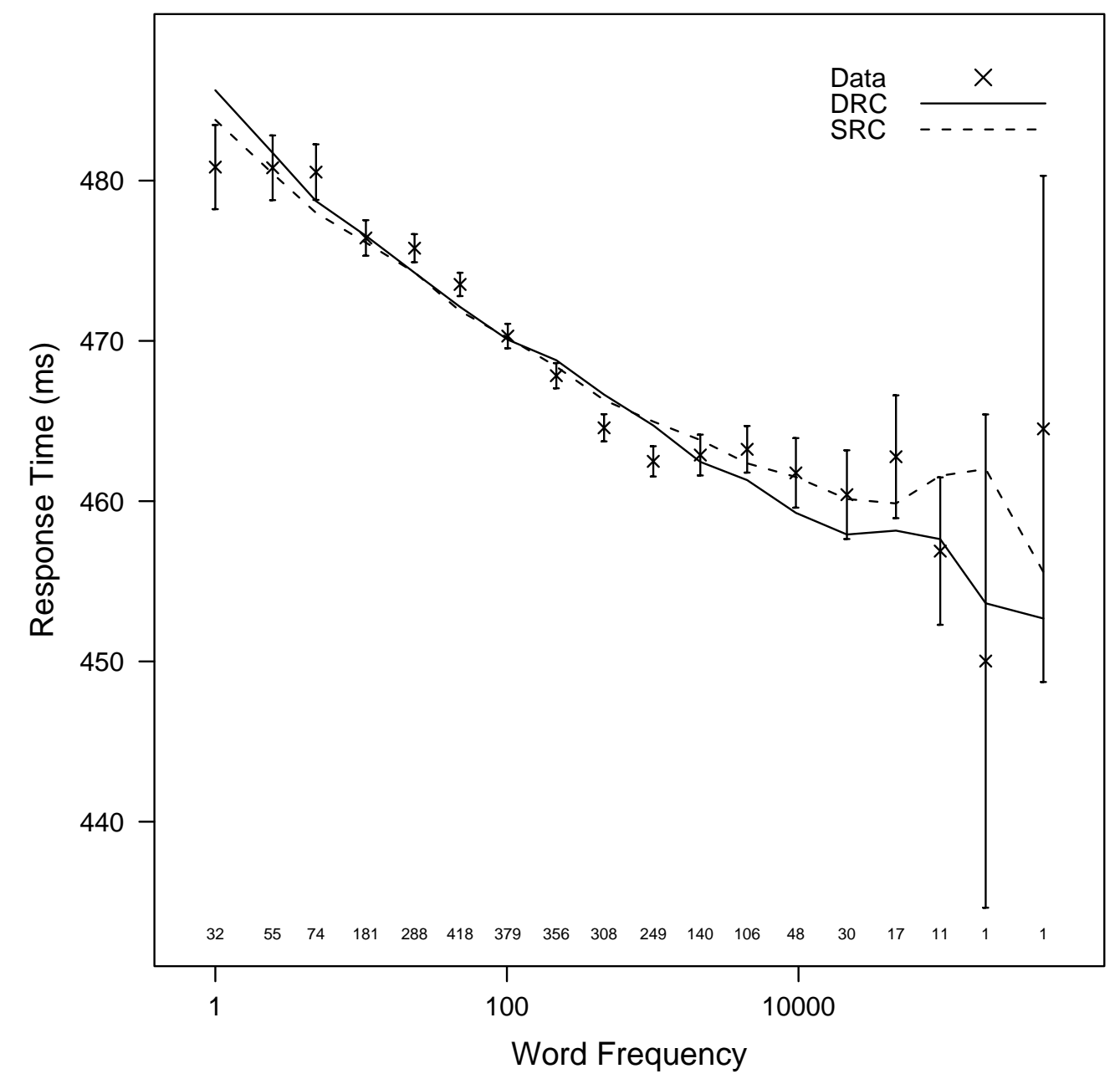




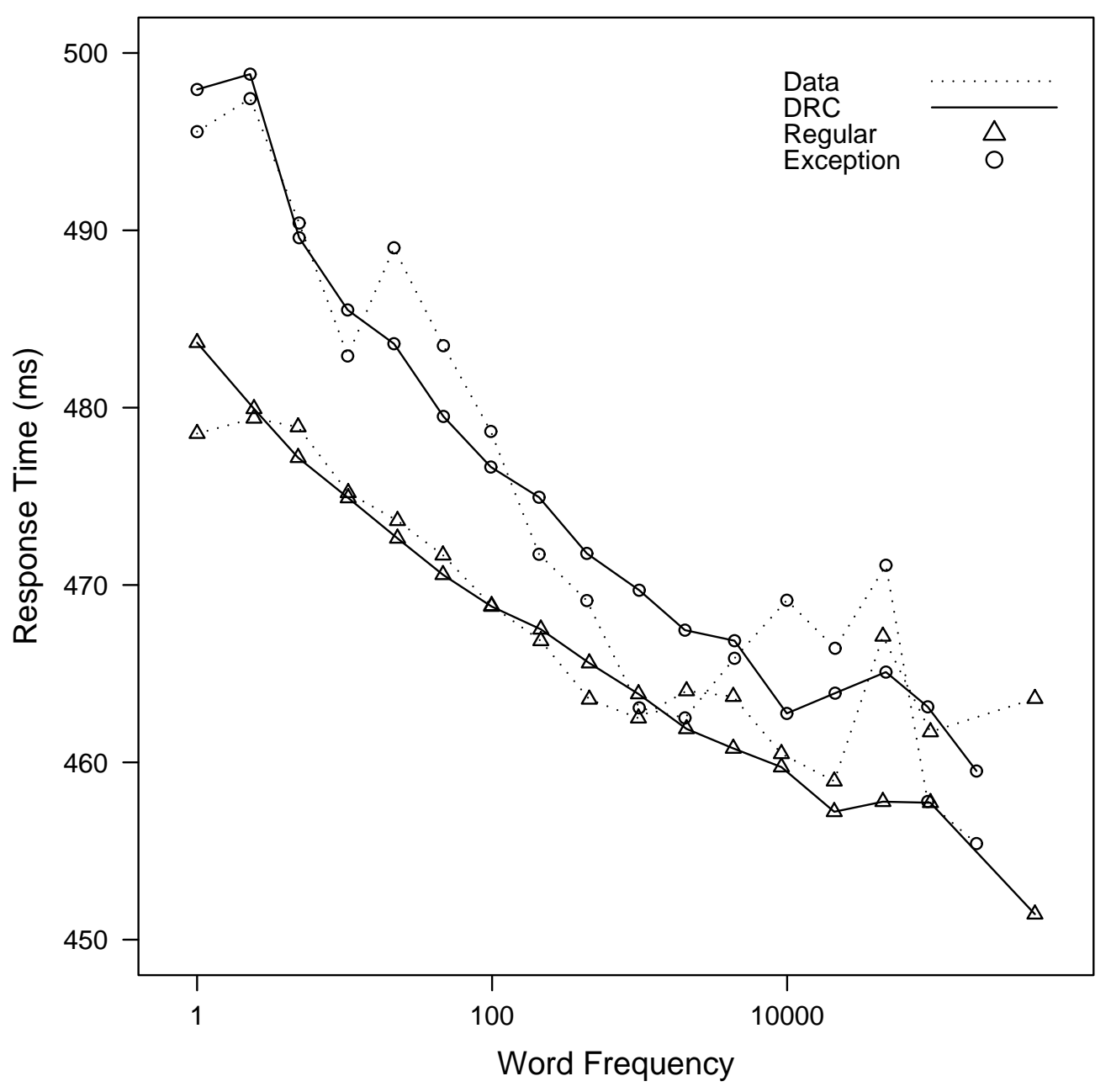




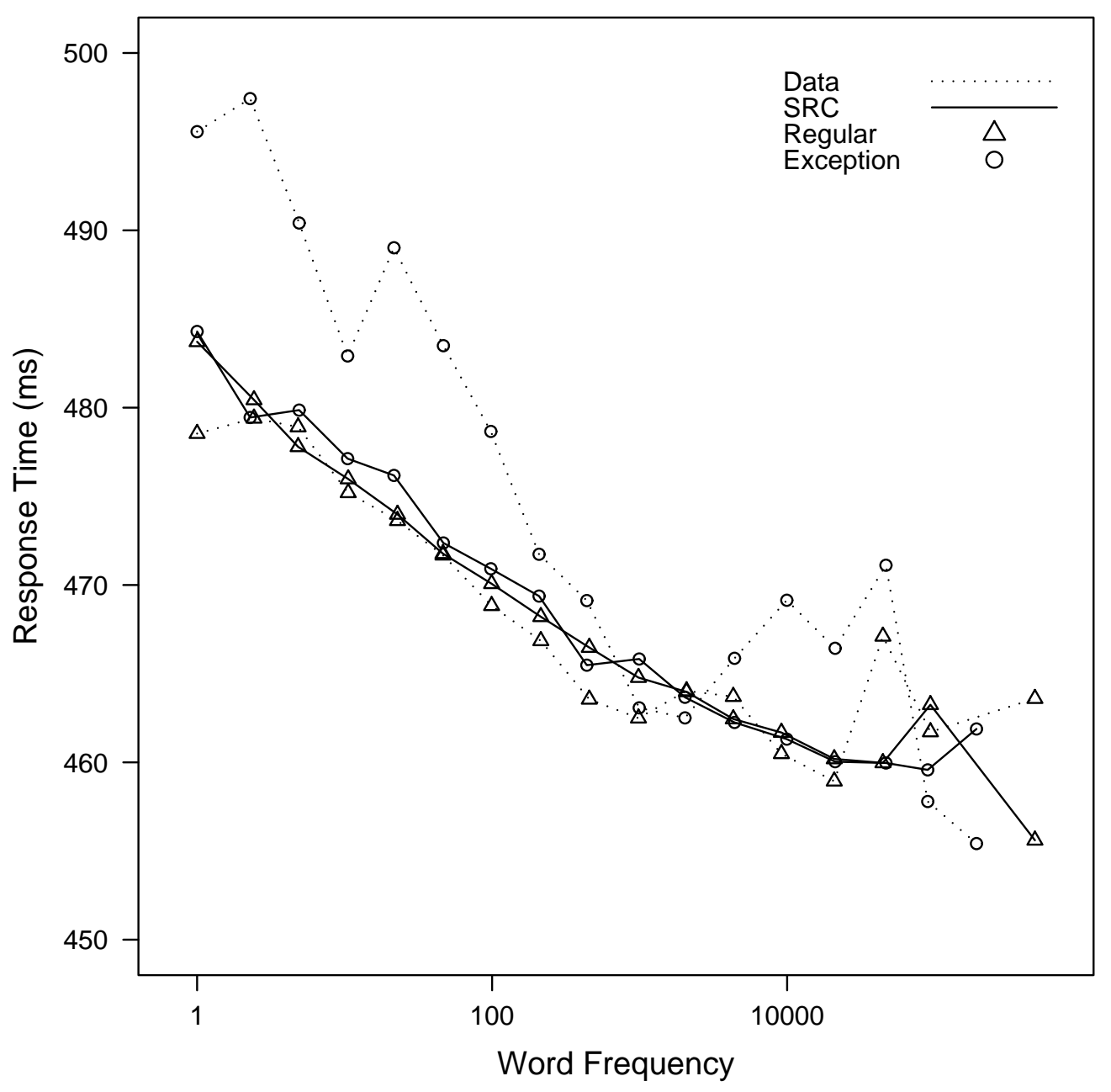




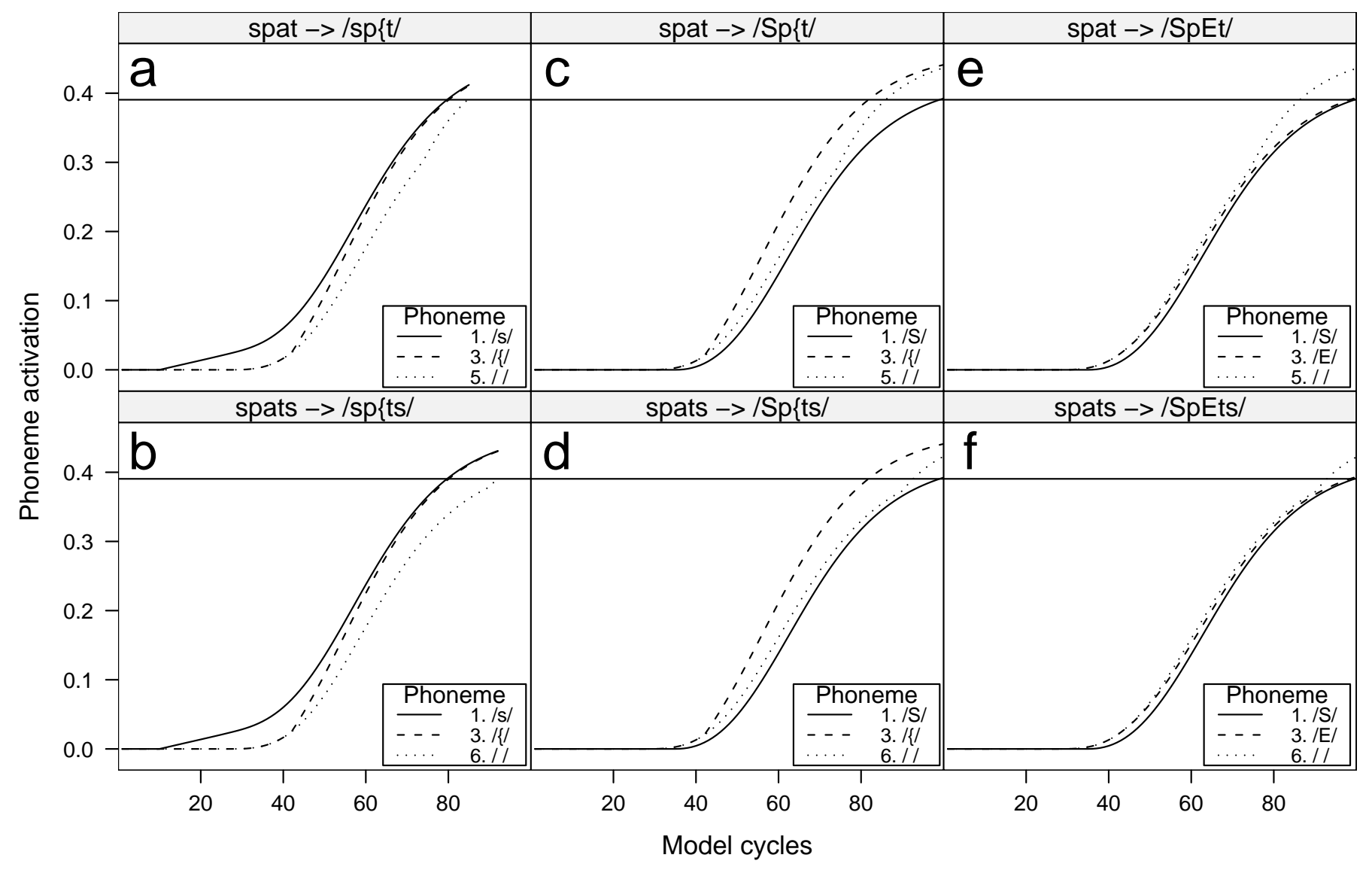

